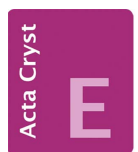

CRYSTALLOGRAPHIC COMMUNICATIONS

ISSN 2056-9890

Received 22 December 2020

Accepted 5 January 2021

Edited by M. Weil, Vienna University of Technology, Austria

Keywords: crystal structure; perimidine; $\pi-\pi$ stacking; hydrogen-bonding; NMR study.

CCDC references: 2051714; 2032890; 2032889

Supporting information: this article has supporting information at journals.iucr.org/e

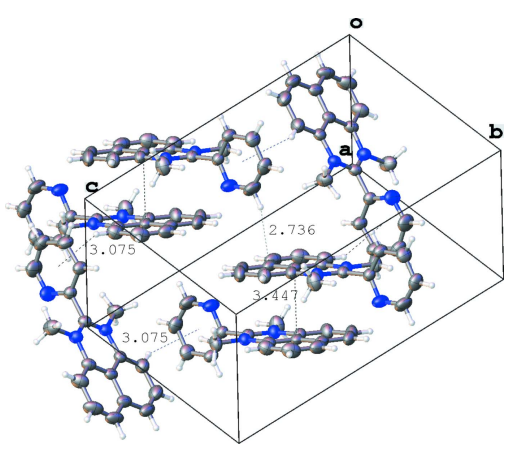

\section{Synthesis and comparative structural study of 2-(pyridin-2-yl)-1H-perimidine and its mono- and di- $N$-methylated analogues}

\author{
Paulina Kalle, ${ }^{a, b}$ Sergei V. Tatarin, ${ }^{a, b}$ Alexander Yu. Zakharov, ${ }^{\text {b Marina A. }}$ \\ Kiseleva $^{\mathrm{a}, \mathrm{b}}$ and Stanislav I. Bezzubov ${ }^{\mathbf{b}}$.
}

aDepartment of Chemistry, Lomonosov Moscow State University, Lenin's Hills, 1-3, Moscow, 119991, Russian Federation, and ${ }^{\mathbf{b}}$ N.S. Kurnakov Institute of General and Inorganic Chemistry, Russian Academy of Sciences, Leninsky pr. 31, Moscow 119991, Russian Federation. *Correspondence e-mail: bezzubov@igic.ras.ru

The title compounds, 2-(pyridin-2-yl)- $1 H$-perimidine $\left(\mathrm{C}_{16} \mathrm{H}_{11} \mathrm{~N}_{3} ; \mathbf{1}\right)$, 1-methyl-2(pyridin-2-yl)-1H-perimidine $\left(\mathrm{C}_{17} \mathrm{H}_{13} \mathrm{~N}_{3} ; 2\right)$, and 1,3-dimethyl-2-(pyridin-2-yl)$1 \mathrm{H}$-perimidinium iodide $\left(\mathrm{C}_{18} \mathrm{H}_{16} \mathrm{~N}_{3}{ }^{+} \cdot \mathrm{I}^{-} ; 3\right)$ were synthesized under mild conditions and their structures were determined by ${ }^{1} \mathrm{H}$ NMR spectroscopy and single-crystal X-ray analysis. The $N$-methylation of the nitrogen atom $(s)$ at the perimidine moiety results in a significant increase of the interplane angle between the pyridin-2-yl ring and the perimidine system. The unsubstituted perimidine (1) forms a weak intramolecular $\mathrm{N}-\mathrm{H} \cdots \mathrm{N}$ bond that consolidates the molecular conformation. In the crystal structures of $\mathbf{1}-\mathbf{3}$, the molecular entities all are assembled through $\pi-\pi$ and $\mathrm{C}-\mathrm{H} \cdots \pi$ interactions.

\section{Chemical context}

Perimidines are fused nitrogen heterocyclic aromatics possessing equally a $\pi$-electron excess and a $\pi$-electron deficiency that determine their diverse reactivities as well as their unique optical and spectroscopic properties (Pozharskii et al., 2020). These compounds have attracted considerable attention over the past two decades because of their growing application in industrial chemistry (especially as dyes and pigments), as optoelectronics, in biotechnology and medicinal chemistry (Sahiba \& Agarwal, 2020).

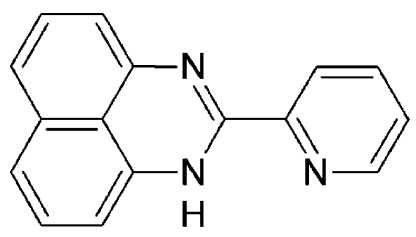

1<smiles>CN1C(c2ccccn2)=Nc2cccc3cccc1c23</smiles>

2<smiles></smiles>

3

Herein, we report structural studies of 1- $H$-2-(pyridin-2yl)perimidine (1) and its mono- and di- $N$-methylated analogues ( $\mathbf{2}$ and $\mathbf{3}$, respectively). 


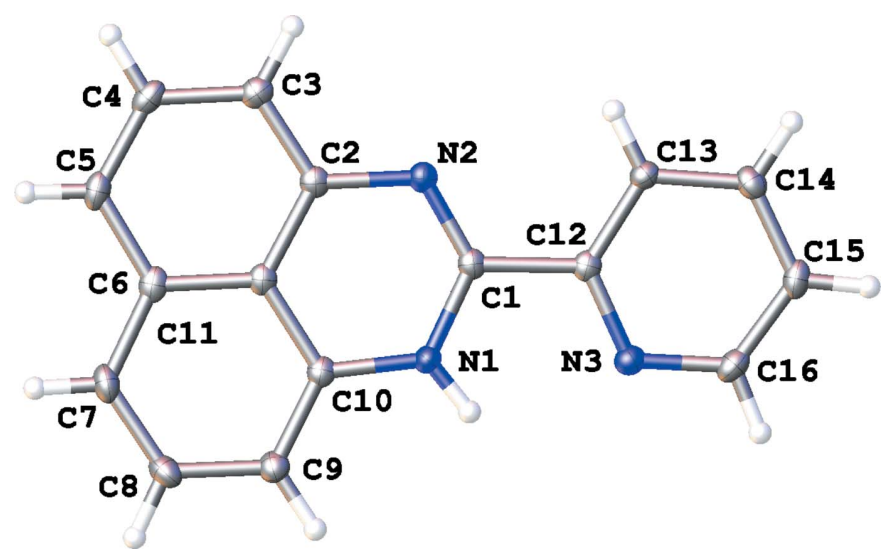

Figure 1

The molecular structure of 1-H-2-(pyridin-2-yl)perimidine (1), with displacement ellipsoids drawn at the $50 \%$ probability level.

\section{Structural commentary}

The compositions and structures of the synthesized compounds were determined both by ${ }^{1} \mathrm{H}$ NMR spectroscopy (for assignment, see: Figs. S1-S3 in the supporting information) and single-crystal X-ray analysis. In all cases, the organic molecules occupy general positions and comprise an essentially flat perimidine system and the pyridyl ring. Depending on the number of $N$-substituents, the ring systems are twisted to a greater or lesser extent (Figs. 1-3). The unsubstituted molecule of $\mathbf{1}$ is almost planar with the dihedral angle between the aromatic parts as small as $1.60(5)^{\circ}$, while the molecules of $\mathbf{2}$ and especially $\mathbf{3}$ show notably larger interplane angles [59.39 (8) and $87.21(9)^{\circ}$, respectively] because of steric repulsion between the $N$-methyl group(s) and the pyridin-2-yl ring. The flat conformation of $\mathbf{1}$ may be stabilized by a weak intramolecular hydrogen bond between the perimidine N1$\mathrm{H} 1$ donor group and the pyridyl N3 acceptor group $[d(\mathrm{~N} 1 \cdots \mathrm{N} 3)=2.626(2) \AA, d(\mathrm{~N} 1-\mathrm{H} 1)=0.87(2) \AA$, $\left.d(\mathrm{H} 1 \cdots \mathrm{N} 3)=2.19(2) \AA, \quad \mathrm{N} 1-\mathrm{H} 1 \cdots \mathrm{N} 3=110.9(17)^{\circ}\right]$ whereas in the molecular structure of $\mathbf{2}$ the pyridyl nitrogen

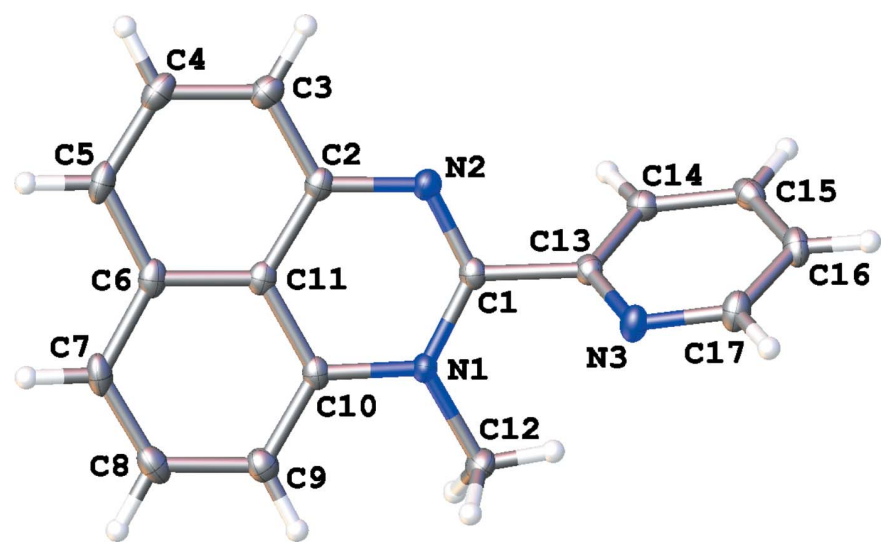

Figure 2

The molecular structure of 1-methyl-2-(pyridin-2-yl)perimidine (2), with displacement ellipsoids drawn at the $50 \%$ probability level.

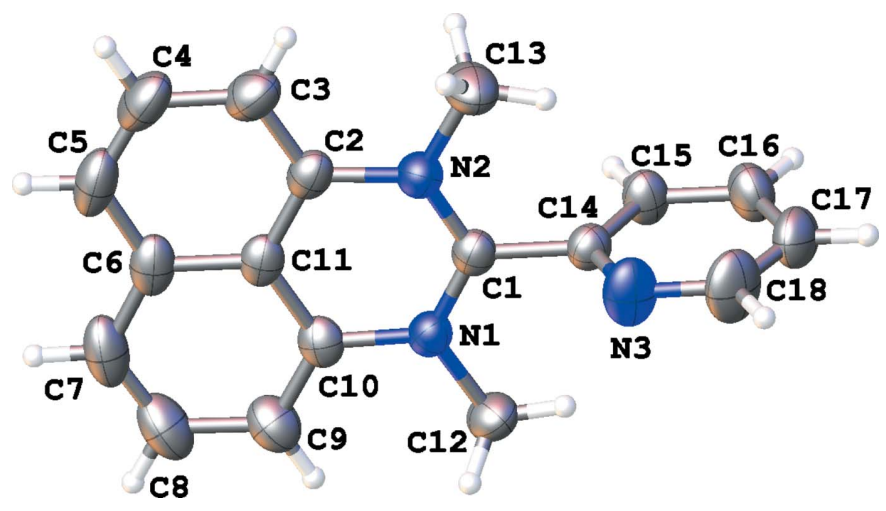

Figure 3

The molecular structure of 1,3-dimethyl-2-(pyridin-2-yl)perimidinium iodide (3, only the cation is presented), with displacement ellipsoids drawn at the $50 \%$ probability level.

atom participates in a weak intramolecular $\mathrm{C} 12\left(s p^{3}\right)-$ $\mathrm{H} 12 A \cdots \mathrm{N} 3$ contact $[d(\mathrm{H} 12 A \cdots \mathrm{N} 3)=2.46(2) \AA ; \mathrm{C} 12 \cdots \mathrm{N} 3=$ 3.059 (1) $\AA$; $\left.\mathrm{C} 12-\mathrm{H} 12 A \cdots \mathrm{N} 3=120.8(18)^{\circ}\right]$. Compound 3 is a salt and its crystal consists of doubly $\mathrm{N}$-methylated perimidinium cations and iodide counter-ions combined mainly through Coulombic interactions.

${ }^{1} \mathrm{H}$ NMR spectroscopic studies of $\mathbf{1}-\mathbf{3}$ revealed correlations between the chemical shifts of some bands in the spectra and the mutual arrangement of the perimidine and pyridyl aromatics. In the ${ }^{1} \mathrm{H}$ NMR spectrum of $\mathbf{1}$ in $\mathrm{CDCl}_{3}$, doublets at 6.36 and $6.91 \mathrm{ppm}$ arise from the $\mathbf{j}$ and $\mathbf{e}$ protons, respectively, while the other protons of the perimidine core appear as complex multiplets in the range $7.06-7.25$ ppm (Fig. S1). A similar set of bands (corresponding to the same protons) with slightly different chemical shifts can be found in the ${ }^{1} \mathrm{H}$ NMR spectrum of 2 (Fig. S2) whereas 1,3-dimethyl-2-(pyridin-2yl)perimidinium iodide (3) demonstrates a reduced number of resonance signals (Fig. S3) because the protons of the fused benzene rings become equivalent. The latter results from the above arrangement of the pyridyl ring almost orthogonal to the perimidine system.

For compound 1, solvent-dependent resonance signals in the ${ }^{1} \mathrm{H}$ NMR spectrum were detected. In DMSO- $d_{6}$ as a solvent (Fig. S4), the characteristic doublets arising from the protons $\mathbf{j}$ and $\mathbf{e}$ are now closer (6.74 and $6.79 \mathrm{ppm}$, respectively) while the integrated intensity of the signal of the $\mathrm{N}-\mathrm{H}$ proton becomes lower $(0.77 \mathrm{ppm})$ which may result from a weakening of the intramolecular $\mathrm{N}-\mathrm{H} \cdots \mathrm{N}$ hydrogen bond by the polar solvent.

\section{Supramolecular features}

In the crystal of $\mathbf{1}$, molecules are assembled through parallel displaced $\pi-\pi$ stacking interactions between the flat pyridyl and perimidine fragments distant by 3.295 (4) $\AA$ (C5 …N1$\left.\mathrm{C} 11_{\text {centroid }}\right)$ and $3.302(4) \AA \quad\left(\mathrm{N} 2 \cdots \mathrm{py}_{\text {centroid }}\right)$, while the resulting offset stacks [centroid-to-centroid shift between the adjacent molecules in the stack $3.791(4) \AA]$ are grafted together in the resulting three-dimensional network by a $\mathrm{C}-$ 


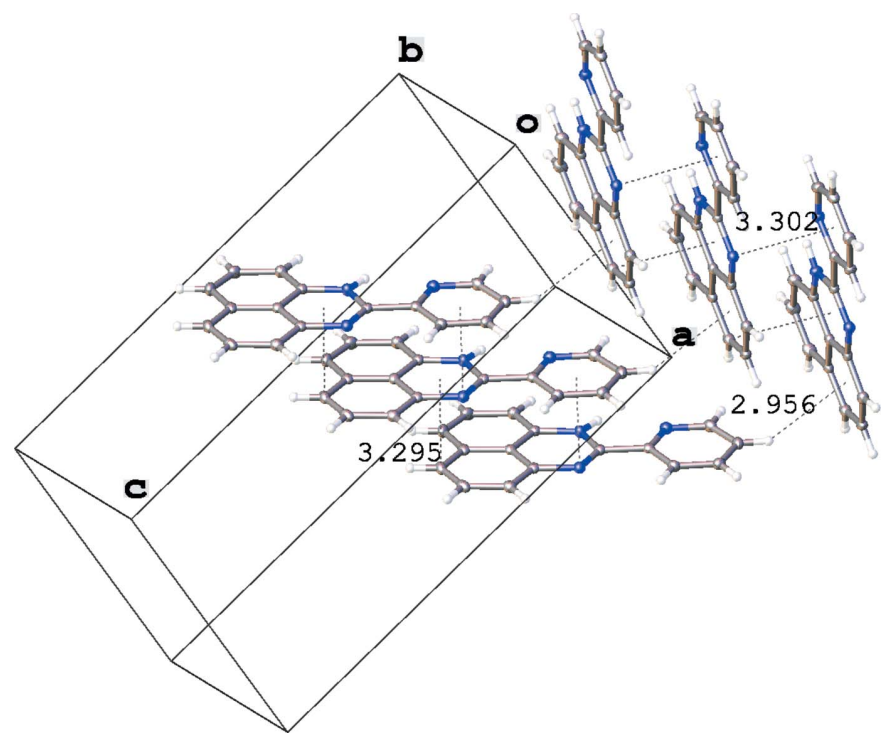

Figure 4

Intermolecular contacts $(\AA)$ in the crystal of $1-H-2$-(pyridin-2-yl)perimidine (1). Displacement ellipsoids are shown at the $50 \%$ probability level.

$\mathrm{H} \cdots \pi$ interaction $[d(\mathrm{H} \cdots \pi)=2.96(2) \AA]$ involving the pyridyl $\mathrm{H} 15$ atom and the centroid of the $\mathrm{C} 2-\mathrm{C} 11$ ring (Fig. 4). In contrast, two types of $\pi-\pi$ interactions are found in the crystal of $\mathbf{2}$, one of which is a slipped stacking [centroid-tocentroid shift $1.645(2) \AA]$ between the perimidine units $\left[d\left(\mathrm{C} 7 \cdots \mathrm{N} 1-\mathrm{C} 11_{\text {centroid }}\right)=3.375(2) \AA, d\left(\mathrm{C} 9 \cdots \mathrm{N} 1-\mathrm{C} 11_{\text {centroid }}\right)\right.$ $=3.774(3) \AA, d\left(\mathrm{C} 11 \cdots \mathrm{C} 6-\mathrm{C} 11_{\text {centroid }}\right)=3.423$ (2) $\AA$ ] while the other is a pyridyl-pyridyl contact [distance between the C16 atom and the pyridyl ring 3.499 (3) A] connecting the stacks together. Intermolecular contacts between the $\mathrm{H} 12 \mathrm{C}$ atom and the C6-C11 ${ }_{\text {centroid }}[3.17$ (2) $\AA$ ] and between the H14 atom and $\mathrm{C} 2-\mathrm{C} 11_{\text {centroid }}[3.684$ (19) $\AA$ ] form a three-dimensional network in the crystal structure of 2 (Fig. 5). In the crystal structure of $\mathbf{3}$, there are $\pi-\pi$-bonded dimers [interplane distance 3.447 (3) $\AA$ between the perimidine moieties], which form dense layers via $\mathrm{C}-\mathrm{H} \cdots \pi$ interactions $[d(\mathrm{H} \cdots \pi)=$ 3.132 (2) $\AA$ between the H18 atom and the centroid of the C6-

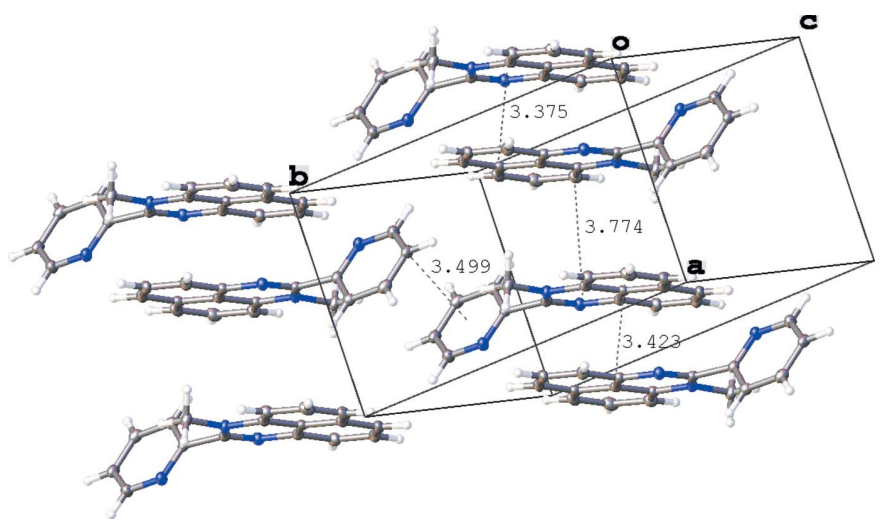

Figure 5

Intermolecular contacts $(\AA)$ in the crystal of 1-methyl-2-(pyridin-2yl)perimidine (2). Displacement ellipsoids are shown at the $50 \%$ probability level.

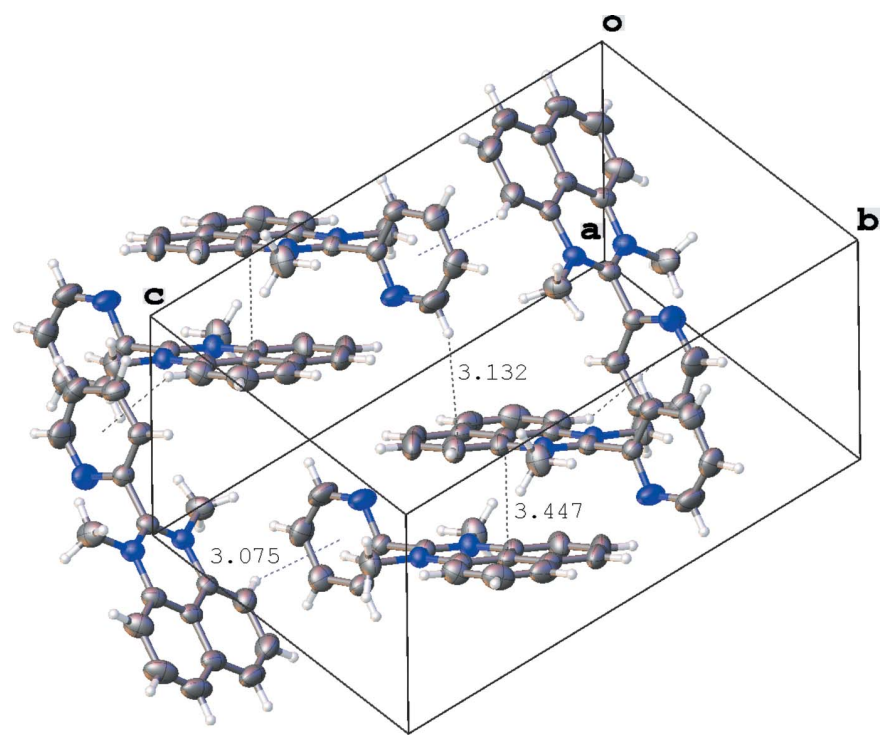

Figure 6

Intermolecular contacts $(\AA)$ in the crystal of 1,3-dimethyl-2-(pyridin-2yl)perimidinium iodide (3, only cations are presented). Displacement ellipsoids are shown at the $50 \%$ probability level.

C11 ring and 3.075 (2) $\AA$ between the H9 atom and the centroid of the pyridyl ring; Fig. 6]. The resulting cationic organic layers and anionic iodide layers alternate along the $c$ axis.

\section{Database survey}

Though many perimidines have been prepared so far, fewer than 60 crystal structures of them (including a few of metal complexes) have been published (Pozharskii et al., 2020; Hill et al., 2018; Bahena et al., 2019; Booysen et al., 2016). Crystal structures of several 1,3-dimethyl-2-arylperimidinium iodides have been determined ( $\mathrm{Li}$ et al., 2017). A comprehensive structural study of 2-arylperimidines (including those having intramolecular hydrogen bonds) has also been conducted (Foces-Foces et al., 1993; Llamas-Saiz et al., 1995).

\section{Synthesis and crystallization}

The title compounds were prepared as follows:

1-H-2-(pyridin-2-yl)perimidine (1).

A mixture of 1,8-diaminonaphthalene $(4.523 \mathrm{~g}, 28.6 \mathrm{mmol})$, pyridin-2-ylcarboxaldehyde $(2.72 \mathrm{ml}, 28.6 \mathrm{mmol})$ and sodium metabisulfite $(16.317 \mathrm{~g}, 85.8 \mathrm{mmol})$ in ethanol $(50 \mathrm{ml})$ was refluxed under Ar for $4 \mathrm{~h}$. The reaction mixture was evaporated to dryness, washed with water and redissolved in ethanol. Keeping the resulting solution in a freezer overnight gave a red powder, which was recrystallized from methylene chloride and dried in vacuo. Yield $6 \mathrm{~g}$ (86\%). Single crystals suitable for X-ray analysis were grown by slow evaporation of the solvent from a solution of the substance in methylene chloride. 
Table 1

Experimental details.

(1)

Crystal data

Chemical formula

$M_{\mathrm{r}}$

Crystal system, space group

Temperature (K)

$a, b, c(\AA)$

$\beta\left({ }^{\circ}\right)$

$V\left(\AA^{3}\right)$

$Z$

Radiation type

$\mu\left(\mathrm{mm}^{-1}\right)$

Crystal size (mm)

Data collection

Diffractometer

Absorption correction

$T_{\min }, T_{\max }$

No. of measured, independent and

observed $[I>2 \sigma(I)]$ reflections

$R_{\text {int }}$

$(\sin \theta / \lambda)_{\max }\left(\AA^{-1}\right)$

$\mathrm{C}_{16} \mathrm{H}_{11} \mathrm{~N}_{3}$

245.28

Monoclinic, $P 2_{1} / c$

100

13.5479 (5), 5.0242 (2), 17.3881 (7)

$101.382(2)$

$1160.28(8)$

4

Mo $K \alpha$

0.09

$0.42 \times 0.1 \times 0.08$

Bruker D8 Venture

Multi-scan ( $S A D A B S$; Krause et al., 2015)

$0.684,0.746$

$16322,2866,2405$

0.036

0.667

$0.057,0.140,1.05$

2866

216

All $\mathrm{H}$-atom parameters refined

$0.39,-0.33$
(2)

$\mathrm{C}_{17} \mathrm{H}_{13} \mathrm{~N}_{3}$
259.30
Monoclinic, $P 2_{1} / c$
100
$7.5095(2), 12.1216(3), 13.5616(4)$
$92.547(1)$
$1233.25(6)$
4
Mo $K \alpha$
0.09
$0.34 \times 0.12 \times 0.11$

$0.34 \times 0.12 \times 0.11$

Bruker D8 Venture

Multi-scan (SADABS; Krause et al., 2015)

$0.685,0.746$

$14029,3280,2803$

0.035

0.682

$0.060,0.146,1.04$

3280

233

All $\mathrm{H}$-atom parameters refined

$0.45,-0.34$
(3)

$\mathrm{C}_{18} \mathrm{H}_{16} \mathrm{~N}_{3}{ }^{+} \cdot \mathrm{I}^{-}$
401.24
Monoclinic, $P 2_{1} / n$
230
$9.8821(2), 9.7125(2), 17.9839(4)$
$103.676(1)$
$1677.15(6)$
4
Mo $K \alpha$
1.91
$0.32 \times 0.18 \times 0.13$

Bruker SMART APEXII

Multi-scan ( $S A D A B S$; Krause et al., 2015)

$0.668,0.746$

$28389,4146,3700$

0.026

0.668

$0.028,0.067,1.06$

4146

201

$\mathrm{H}$-atom parameters constrained $0.72,-0.46$

$\Delta \rho_{\max }, \Delta \rho_{\min }\left(\mathrm{e} \AA^{-3}\right)$

Computer programs: APEX3 and SAINT (Bruker, 2017), SHELXT (Sheldrick, 2015a), SHELXL (Sheldrick, 2015b), OLEX2 (Dolomanov et al., 2009) and publCIF (Westrip, 2010).

${ }^{1} \mathrm{H}$ NMR $\left(\mathrm{CDCl}_{3}, \mathrm{ppm}, 400 \mathrm{MHz}\right): \delta 6.36(d, J=7.4 \mathrm{~Hz}, 1 \mathrm{H}$, $\left.\mathrm{H}_{\text {naph }}\right), 6.91\left(d, J=7.4 \mathrm{~Hz}, 1 \mathrm{H}, \mathrm{H}_{\text {naph }}\right), 7.06-7.25(m, 4 \mathrm{H}$, $\left.\mathrm{H}_{\text {naph }}\right), 7.44-7.47\left(m, 1 \mathrm{H}, \mathrm{H}_{\mathrm{py}}\right), 7.88\left(t d, J_{I}=7.8 \mathrm{~Hz}, J_{2}=1.7 \mathrm{~Hz}\right.$, $\left.1 \mathrm{H}, \mathrm{H}_{\mathrm{py}}\right), 8.44\left(d, J=7.6 \mathrm{~Hz}, 1 \mathrm{H}, \mathrm{H}_{\mathrm{py}}\right), 8.62-8.64\left(m, 1 \mathrm{H}, \mathrm{H}_{\mathrm{py}}\right)$, 9.39 (br. s, 1H, N-H). See supplementary Fig. S1.

1-Methyl-2-(pyridin-2-yl)perimidine (2).

To a mixture of $\mathbf{1}(0.250 \mathrm{~g}, 1.02 \mathrm{mmol})$, solid $\mathrm{KOH}(0.057 \mathrm{~g}$, $1.02 \mathrm{mmol})$ and anhydrous $\mathrm{K}_{2} \mathrm{CO}_{3}(0.141 \mathrm{~g}, 1.02 \mathrm{mmol})$ in anhydrous Ar-saturated acetonitrile methyl iodide $(0.064 \mathrm{ml}$, $1.02 \mathrm{mmol}$ ) was added dropwise upon stirring and the resulting suspension was heated at $323 \mathrm{~K}$ for $3 \mathrm{~h}$ and then at r.t. for two days. The reaction mixture was evaporated to dryness and the crude product was purified by column chromatography (eluent hexane/ethyl acetate $1 / 1 v / v$ ), recrystallized from a mixture of $\mathrm{CH}_{2} \mathrm{Cl}_{2} /$ hexane and dried in vacuo. Yield $185 \mathrm{mg}$ (70\%). Single crystals suitable for X-ray analysis were grown by slow evaporation of the solvent from a solution of the substance in chloroform.

${ }^{1} \mathrm{H}$ NMR $\left(\mathrm{CDCl}_{3}, \mathrm{ppm}, 400 \mathrm{MHz}\right): \delta 3.17\left(s, 3 \mathrm{H}, \mathrm{N}-\mathrm{CH}_{3}\right)$, $6.32\left(d d, J_{1}=7.2 \mathrm{~Hz}, J_{2}=1.0 \mathrm{~Hz}, 1 \mathrm{H}, \mathrm{H}_{\text {naph }}\right), 6.94\left(d d, J_{1}=\right.$ $\left.7.3 \mathrm{~Hz}, J_{2}=1.0 \mathrm{~Hz}, 1 \mathrm{H}, \mathrm{H}_{\text {naph }}\right), 7.17-7.32\left(m, 4 \mathrm{H}, \mathrm{H}_{\text {naph }}\right), 7.39-$ $7.42\left(m, 1 \mathrm{H}, \mathrm{H}_{\mathrm{py}}\right), 7.77-7.80\left(m, 1 \mathrm{H}, \mathrm{H}_{\mathrm{py}}\right), 7.86-7.89(m, 1 \mathrm{H}$, $\left.\mathrm{H}_{\mathrm{py}}\right), 8.70\left(m, 1 \mathrm{H}, \mathrm{H}_{\mathrm{py}}\right)$. See supplementary Fig. S2.

1,3-Dimethyl-2-(pyridin-2-yl)perimidinium iodide (3).

This compound was isolated from the above reaction mixture (synthesis of compound 2 ) as a side product $(15 \mathrm{mg}$ ). Single crystals suitable for X-ray analysis were grown by slow evaporation of the solvent from a solution of the substance in ethanol.
${ }^{1} \mathrm{H}$ NMR $\left(\mathrm{CDCl}_{3}, \mathrm{ppm}, 400 \mathrm{MHz}\right): \delta 3.34\left(s, 6 \mathrm{H}, \mathrm{N}-\mathrm{CH}_{3}\right)$, $6.96\left(d, J=7.7 \mathrm{~Hz}, 2 \mathrm{H}, \mathrm{H}_{\text {naph }}\right), 7.50\left(m, 2 \mathrm{H}, \mathrm{H}_{\text {naph }}\right), 7.60(m$, $\left.2 \mathrm{H}, \mathrm{H}_{\text {naph }}\right), 7.66-7.70\left(m, 1 \mathrm{H}, \mathrm{H}_{\mathrm{py}}\right), 8.19\left(t d, J_{I}=7.8 \mathrm{~Hz}, J_{2}=\right.$ $\left.1.7 \mathrm{~Hz}, 1 \mathrm{H}, \mathrm{H}_{\mathrm{py}}\right), 8.65-8.68\left(m, 1 \mathrm{H}, \mathrm{H}_{\mathrm{py}}\right), 9.24-9.26(m, 1 \mathrm{H}$, $\left.\mathrm{H}_{\mathrm{py}}\right)$. See supplementary Fig. S3.

\section{Refinement}

Crystal data, data collection and structure refinement details are summarized in Table 1. Hydrogen atoms in the structures of $\mathbf{1}$ and $\mathbf{2}$ were located from difference electron density maps and were refined freely. In the structure of $\mathbf{3}$, hydrogen atoms were placed in calculated positions and refined using a riding model $\left[\mathrm{C}-\mathrm{H}=0.94-0.97 \AA\right.$ with $\left.U_{\text {iso }}(\mathrm{H})=1.2-1.5 U_{\text {eq }}(\mathrm{C})\right]$.

\section{Acknowledgements}

$\mathrm{X}$-ray diffraction studies were performed at the Centre of Shared Equipment of IGIC RAS. Dr I. M. Vatsouro is acknowledged for assistance with the NMR measurements.

\section{Funding information}

Funding for this research was provided by: Presidential Grant Program (grant No. MK-1200.2020.3).

\section{References}

Bahena, E. N., Gijon, C. A. F., Fomine, S., Alexandrova, L. \& Le Lagadec, R. (2019). Eur. J. Inorg. Chem. 3494-3502. 
Booysen, I. N., Ebinumoliseh, I., Sithebe, S., Akerman, M. P. \& Xulu, B. (2016). Polyhedron, 117, 755-760.

Bruker (2017). APEX3 and SAINT. Bruker AXS Inc., Madison, Wisconsin, USA.

Dolomanov, O. V., Bourhis, L. J., Gildea, R. J., Howard, J. A. K. \& Puschmann, H. (2009). J. Appl. Cryst. 42, 339-341.

Foces-Foces, C., Llamas-Saiz, A. L., Claramunt, R. M., Sanz, D., Dotor, J. \& Elguero, J. (1993). J. Cryst. Spec. Res. 23, 305-312.

Hill, A. F., Ma, C., McQueen, C. M. A. \& Ward, J. S. (2018). Dalton Trans. 47, 1577-1587.

Krause, L., Herbst-Irmer, R., Sheldrick, G. M. \& Stalke, D. (2015). J. Appl. Cryst. 48, 3-10.
Li, Z.-Y., Zhang, M., Yuan, X.-Y. \& Yuan, L. (2017). Z. Kristallogr. New Cryst. Struct. 232, 429-430.

Llamas-Saiz, A. L., Foces-Foces, C., Sanz, D., Claramunt, R. M., Dotor, J., Elguero, J., Catalan, J. \& del Valle, J. C. (1995). J. Chem. Soc., Perkin Trans. 2, 1389-1398.

Pozharskii, A. F., Gulevskaya, A. V., Claramunt, R. M., Alkorta, I. \& Elguero, J. (2020). Russ. Chem. Rev. 89, 1204-1260.

Sahiba, N. \& Agarwal, S. (2020). Top. Curr. Chem. 378 article number 44.

Sheldrick, G. M. (2015a). Acta Cryst. A71, 3-8.

Sheldrick, G. M. (2015b). Acta Cryst. C71, 3-8.

Westrip, S. P. (2010). J. Appl. Cryst. 43, 920-925. 


\section{supporting information}

Acta Cryst. (2021). E77, 96-100 [https://doi.org/10.1107/S205698902100013X]

Synthesis and comparative structural study of 2-(pyridin-2-yl)-1H-perimidine and its mono- and di- $\mathrm{N}$-methylated analogues

Paulina Kalle, Sergei V. Tatarin, Alexander Yu. Zakharov, Marina A. Kiseleva and Stanislav I. Bezzubov

Computing details

For all structures, data collection: APEX3 (Bruker, 2017); cell refinement: SAINT (Bruker, 2017); data reduction: SAINT (Bruker, 2017); program(s) used to solve structure: SHELXT (Sheldrick, 2015a); program(s) used to refine structure: SHELXL (Sheldrick, 2015b); molecular graphics: OLEX2 (Dolomanov et al., 2009); software used to prepare material for publication: publCIF (Westrip, 2010).

2-(Pyridin-2-yl)-1H-perimidine (1)

Crystal data

$\mathrm{C}_{16} \mathrm{H}_{11} \mathrm{~N}_{3}$

$M_{r}=245.28$

Monoclinic, $P 2_{1} / c$

$a=13.5479(5) \AA$

$b=5.0242(2) \AA$

$c=17.3881(7) \AA$

$\beta=101.382(2)^{\circ}$

$V=1160.28(8) \AA^{3}$

$Z=4$

\section{Data collection}

Bruker D8 Venture diffractometer

Radiation source: microfocus sealed X-ray tube, Incoatec $\mathrm{I} \mu \mathrm{S}$ microsource

Focusing mirrors monochromator Detector resolution: 10.4 pixels $\mathrm{mm}^{-1}$ $\omega$-scan

Absorption correction: multi-scan

(SADABS; Krause et al., 2015)

\section{Refinement}

Refinement on $F^{2}$

Least-squares matrix: full

$R\left[F^{2}>2 \sigma\left(F^{2}\right)\right]=0.057$

$w R\left(F^{2}\right)=0.140$

$S=1.05$

2866 reflections

216 parameters

0 restraints
$F(000)=512$

$D_{\mathrm{x}}=1.404 \mathrm{Mg} \mathrm{m}^{-3}$

Mo $K \alpha$ radiation, $\lambda=0.71073 \AA$

Cell parameters from 7163 reflections

$\theta=3.1-28.3^{\circ}$

$\mu=0.09 \mathrm{~mm}^{-1}$

$T=100 \mathrm{~K}$

Needle, red

$0.42 \times 0.1 \times 0.08 \mathrm{~mm}$

$T_{\min }=0.684, T_{\max }=0.746$

16322 measured reflections

2866 independent reflections

2405 reflections with $I>2 \sigma(I)$

$R_{\text {int }}=0.036$

$\theta_{\max }=28.3^{\circ}, \theta_{\min }=3.5^{\circ}$

$h=-18 \rightarrow 18$

$k=-6 \rightarrow 6$

$l=-23 \rightarrow 22$

Primary atom site location: dual

Hydrogen site location: difference Fourier map

All $\mathrm{H}$-atom parameters refined

$w=1 /\left[\sigma^{2}\left(F_{\mathrm{o}}^{2}\right)+(0.057 P)^{2}+1.1159 P\right]$

where $P=\left(F_{\mathrm{o}}^{2}+2 F_{\mathrm{c}}^{2}\right) / 3$

$(\Delta / \sigma)_{\max }<0.001$

$\Delta \rho_{\max }=0.39 \mathrm{e} \AA^{-3}$

$\Delta \rho_{\min }=-0.33$ e $\AA^{-3}$ 


\section{Special details}

Geometry. All esds (except the esd in the dihedral angle between two 1.s. planes) are estimated using the full covariance matrix. The cell esds are taken into account individually in the estimation of esds in distances, angles and torsion angles; correlations between esds in cell parameters are only used when they are defined by crystal symmetry. An approximate (isotropic) treatment of cell esds is used for estimating esds involving l.s. planes.

Fractional atomic coordinates and isotropic or equivalent isotropic displacement parameters $\left(\AA^{2}\right)$

\begin{tabular}{lllll}
\hline & $x$ & $y$ & $z$ & $U_{\text {iso }} * / U_{\text {eq }}$ \\
\hline N1 & $0.67572(10)$ & $0.4402(3)$ & $0.27627(8)$ & $0.0154(3)$ \\
N3 & $0.71391(10)$ & $0.0786(3)$ & $0.17704(8)$ & $0.0168(3)$ \\
N2 & $0.84493(10)$ & $0.5837(3)$ & $0.30290(8)$ & $0.0155(3)$ \\
C12 & $0.79419(12)$ & $0.2223(3)$ & $0.21158(9)$ & $0.0143(3)$ \\
C10 & $0.64357(12)$ & $0.6204(3)$ & $0.32655(9)$ & $0.0149(3)$ \\
C1 & $0.77359(12)$ & $0.4307(3)$ & $0.26757(9)$ & $0.0142(3)$ \\
C2 & $0.81956(12)$ & $0.7734(3)$ & $0.35506(9)$ & $0.0150(3)$ \\
C13 & $0.89068(12)$ & $0.1825(3)$ & $0.19733(9)$ & $0.0156(3)$ \\
C3 & $0.89194(13)$ & $0.9430(3)$ & $0.39534(9)$ & $0.0174(3)$ \\
C8 & $0.52046(13)$ & $0.8239(4)$ & $0.38981(10)$ & $0.0200(4)$ \\
C16 & $0.72925(13)$ & $-0.1115(3)$ & $0.12658(10)$ & $0.0189(4)$ \\
C15 & $0.82269(13)$ & $-0.1659(3)$ & $0.10900(9)$ & $0.0191(4)$ \\
C9 & $0.54570(12)$ & $0.6318(3)$ & $0.33781(9)$ & $0.0182(3)$ \\
C6 & $0.69204(12)$ & $0.9893(3)$ & $0.41877(9)$ & $0.0168(3)$ \\
C5 & $0.76845(13)$ & $1.1625(3)$ & $0.45756(9)$ & $0.0186(3)$ \\
C14 & $0.90460(13)$ & $-0.0168(3)$ & $0.14534(10)$ & $0.0181(3)$ \\
C11 & $0.71827(12)$ & $0.7951(3)$ & $0.36697(9)$ & $0.0145(3)$ \\
C7 & $0.59039(13)$ & $0.9992(4)$ & $0.42875(10)$ & $0.0201(4)$ \\
C4 & $0.86549(13)$ & $1.1376(3)$ & $0.44599(9)$ & $0.0187(4)$ \\
H3 & $0.9603(15)$ & $0.931(4)$ & $0.3862(11)$ & $0.020(5)^{*}$ \\
H13 & $0.9452(16)$ & $0.282(4)$ & $0.2226(12)$ & $0.024(5)^{*}$ \\
H8 & $0.4528(16)$ & $0.829(4)$ & $0.3965(12)$ & $0.024(5)^{*}$ \\
H14 & $0.9698(17)$ & $-0.050(5)$ & $0.1362(13)$ & $0.031(6)^{*}$ \\
H9 & $0.4957(14)$ & $0.510(4)$ & $0.3102(11)$ & $0.016(5)^{*}$ \\
H7 & $0.5720(16)$ & $1.125(5)$ & $0.4625(13)$ & $0.030(6)^{*}$ \\
H4 & $0.9168(15)$ & $1.252(4)$ & $0.4733(12)$ & $0.023(5)^{*}$ \\
H16 & $0.6709(15)$ & $-0.203(4)$ & $0.1022(12)$ & $0.024(5)^{*}$ \\
H1 & $0.6349(17)$ & $0.326(5)$ & $0.2490(13)$ & $0.032(6)^{*}$ \\
H5 & $0.7526(14)$ & $1.292(4)$ & $0.4910(12)$ & $0.019(5)^{*}$ \\
H15 & $0.8299(15)$ & $-0.302(4)$ & $0.0730(12)$ & $0.024(5)^{*}$ \\
& & & &
\end{tabular}

Atomic displacement parameters $\left(\AA^{2}\right)$

\begin{tabular}{lllllll}
\hline & $U^{11}$ & $U^{22}$ & $U^{33}$ & $U^{12}$ & $U^{13}$ & $U^{23}$ \\
\hline $\mathrm{N} 1$ & $0.0149(6)$ & $0.0159(7)$ & $0.0152(6)$ & $-0.0011(5)$ & $0.0022(5)$ & $-0.0026(5)$ \\
$\mathrm{N} 3$ & $0.0164(6)$ & $0.0168(7)$ & $0.0166(7)$ & $-0.0005(5)$ & $0.0016(5)$ & $0.0003(5)$ \\
$\mathrm{N} 2$ & $0.0172(6)$ & $0.0147(7)$ & $0.0147(6)$ & $0.0004(5)$ & $0.0033(5)$ & $0.0008(5)$ \\
C12 & $0.0176(7)$ & $0.0129(7)$ & $0.0120(7)$ & $0.0013(6)$ & $0.0018(6)$ & $0.0024(6)$ \\
C10 & $0.0172(7)$ & $0.0141(7)$ & $0.0128(7)$ & $0.0016(6)$ & $0.0016(6)$ & $0.0016(6)$
\end{tabular}


supporting information

\begin{tabular}{lllllll} 
C1 & $0.0169(7)$ & $0.0127(7)$ & $0.0130(7)$ & $0.0016(6)$ & $0.0030(6)$ & $0.0023(6)$ \\
C2 & $0.0183(8)$ & $0.0132(7)$ & $0.0135(7)$ & $0.0008(6)$ & $0.0034(6)$ & $0.0034(6)$ \\
C13 & $0.0154(7)$ & $0.0155(8)$ & $0.0150(7)$ & $-0.0010(6)$ & $0.0011(6)$ & $-0.0002(6)$ \\
C3 & $0.0194(8)$ & $0.0166(8)$ & $0.0158(7)$ & $-0.0012(6)$ & $0.0022(6)$ & $0.0024(6)$ \\
C8 & $0.0166(8)$ & $0.0262(9)$ & $0.0177(8)$ & $0.0044(7)$ & $0.0047(6)$ & $0.0012(7)$ \\
C16 & $0.0223(8)$ & $0.0160(8)$ & $0.0165(8)$ & $-0.0024(7)$ & $-0.0010(6)$ & $-0.0006(6)$ \\
C15 & $0.0284(9)$ & $0.0142(8)$ & $0.0143(7)$ & $0.0027(6)$ & $0.0031(6)$ & $-0.0008(6)$ \\
C9 & $0.0177(8)$ & $0.0197(8)$ & $0.0165(8)$ & $0.0013(6)$ & $0.0017(6)$ & $-0.0005(6)$ \\
C6 & $0.0240(8)$ & $0.0141(7)$ & $0.0118(7)$ & $0.0020(6)$ & $0.0026(6)$ & $0.0025(6)$ \\
C5 & $0.0288(9)$ & $0.0132(8)$ & $0.0135(7)$ & $0.0013(7)$ & $0.0035(6)$ & $-0.0006(6)$ \\
C14 & $0.0181(8)$ & $0.0200(8)$ & $0.0167(8)$ & $0.0042(6)$ & $0.0044(6)$ & $0.0019(6)$ \\
C11 & $0.0186(8)$ & $0.0129(7)$ & $0.0121(7)$ & $0.0014(6)$ & $0.0030(6)$ & $0.0026(6)$ \\
C7 & $0.0248(9)$ & $0.0202(8)$ & $0.0162(8)$ & $0.0063(7)$ & $0.0059(6)$ & $-0.0017(7)$ \\
C4 & $0.0260(8)$ & $0.0148(8)$ & $0.0137(7)$ & $-0.0047(7)$ & $-0.0002(6)$ & $0.0009(6)$ \\
& & & & & & \\
\hline
\end{tabular}

Geometric parameters $\left(A,{ }^{\circ}\right)$

\begin{tabular}{|c|c|c|c|}
\hline $\mathrm{N} 1-\mathrm{C} 10$ & $1.387(2)$ & $\mathrm{C} 8-\mathrm{C} 9$ & $1.410(2)$ \\
\hline $\mathrm{N} 1-\mathrm{C} 1$ & $1.365(2)$ & $\mathrm{C} 8-\mathrm{C} 7$ & $1.371(2)$ \\
\hline $\mathrm{N} 1-\mathrm{H} 1$ & $0.87(2)$ & $\mathrm{C} 8-\mathrm{H} 8$ & $0.95(2)$ \\
\hline $\mathrm{N} 3-\mathrm{C} 12$ & $1.344(2)$ & $\mathrm{C} 16-\mathrm{C} 15$ & $1.387(2)$ \\
\hline $\mathrm{N} 3-\mathrm{C} 16$ & $1.341(2)$ & $\mathrm{C} 16-\mathrm{H} 16$ & $0.94(2)$ \\
\hline $\mathrm{N} 2-\mathrm{C} 1$ & $1.292(2)$ & $\mathrm{C} 15-\mathrm{C} 14$ & $1.384(2)$ \\
\hline $\mathrm{N} 2-\mathrm{C} 2$ & $1.404(2)$ & $\mathrm{C} 15-\mathrm{H} 15$ & $0.94(2)$ \\
\hline $\mathrm{C} 12-\mathrm{C} 1$ & $1.493(2)$ & C9-H9 & $0.97(2)$ \\
\hline $\mathrm{C} 12-\mathrm{C} 13$ & $1.392(2)$ & $\mathrm{C} 6-\mathrm{C} 5$ & $1.417(2)$ \\
\hline $\mathrm{C} 10-\mathrm{C} 9$ & $1.379(2)$ & $\mathrm{C} 6-\mathrm{C} 11$ & $1.420(2)$ \\
\hline $\mathrm{C} 10-\mathrm{C} 11$ & $1.417(2)$ & $\mathrm{C} 6-\mathrm{C} 7$ & $1.422(2)$ \\
\hline $\mathrm{C} 2-\mathrm{C} 3$ & $1.381(2)$ & $\mathrm{C} 5-\mathrm{C} 4$ & $1.374(2)$ \\
\hline $\mathrm{C} 2-\mathrm{C} 11$ & $1.432(2)$ & $\mathrm{C} 5-\mathrm{H} 5$ & $0.93(2)$ \\
\hline $\mathrm{C} 13-\mathrm{C} 14$ & $1.387(2)$ & $\mathrm{C} 14-\mathrm{H} 14$ & $0.94(2)$ \\
\hline $\mathrm{C} 13-\mathrm{H} 13$ & $0.93(2)$ & $\mathrm{C} 7-\mathrm{H} 7$ & $0.93(2)$ \\
\hline $\mathrm{C} 3-\mathrm{C} 4$ & $1.409(2)$ & $\mathrm{C} 4-\mathrm{H} 4$ & $0.95(2)$ \\
\hline $\mathrm{C} 3-\mathrm{H} 3$ & $0.97(2)$ & & \\
\hline $\mathrm{C} 10-\mathrm{N} 1-\mathrm{H} 1$ & $122.0(15)$ & $\mathrm{N} 3-\mathrm{C} 16-\mathrm{H} 16$ & $114.7(13)$ \\
\hline $\mathrm{C} 1-\mathrm{N} 1-\mathrm{C} 10$ & $121.71(14)$ & $\mathrm{C} 15-\mathrm{C} 16-\mathrm{H} 16$ & $121.8(13)$ \\
\hline $\mathrm{C} 1-\mathrm{N} 1-\mathrm{H} 1$ & $116.3(15)$ & $\mathrm{C} 16-\mathrm{C} 15-\mathrm{H} 15$ & $120.4(12)$ \\
\hline $\mathrm{C} 16-\mathrm{N} 3-\mathrm{C} 12$ & $117.31(14)$ & $\mathrm{C} 14-\mathrm{C} 15-\mathrm{C} 16$ & $118.52(15)$ \\
\hline $\mathrm{C} 1-\mathrm{N} 2-\mathrm{C} 2$ & $117.11(14)$ & $\mathrm{C} 14-\mathrm{C} 15-\mathrm{H} 15$ & $121.0(12)$ \\
\hline $\mathrm{N} 3-\mathrm{C} 12-\mathrm{C} 1$ & $115.40(14)$ & $\mathrm{C} 10-\mathrm{C} 9-\mathrm{C} 8$ & $118.76(15)$ \\
\hline $\mathrm{N} 3-\mathrm{C} 12-\mathrm{C} 13$ & $123.28(15)$ & $\mathrm{C} 10-\mathrm{C} 9-\mathrm{H} 9$ & $120.1(11)$ \\
\hline $\mathrm{C} 13-\mathrm{C} 12-\mathrm{C} 1$ & $121.31(14)$ & $\mathrm{C} 8-\mathrm{C} 9-\mathrm{H} 9$ & $121.1(11)$ \\
\hline $\mathrm{N} 1-\mathrm{C} 10-\mathrm{C} 11$ & $115.76(14)$ & $\mathrm{C} 5-\mathrm{C} 6-\mathrm{C} 11$ & $118.24(15)$ \\
\hline $\mathrm{C} 9-\mathrm{C} 10-\mathrm{N} 1$ & $123.16(15)$ & $\mathrm{C} 5-\mathrm{C} 6-\mathrm{C} 7$ & $123.66(15)$ \\
\hline $\mathrm{C} 9-\mathrm{C} 10-\mathrm{C} 11$ & $121.09(15)$ & $\mathrm{C} 11-\mathrm{C} 6-\mathrm{C} 7$ & $118.11(15)$ \\
\hline $\mathrm{N} 1-\mathrm{C} 1-\mathrm{C} 12$ & $114.06(14)$ & $\mathrm{C} 6-\mathrm{C} 5-\mathrm{H} 5$ & $119.6(12)$ \\
\hline $\mathrm{N} 2-\mathrm{C} 1-\mathrm{N} 1$ & $125.35(15)$ & $\mathrm{C} 4-\mathrm{C} 5-\mathrm{C} 6$ & $120.29(15)$ \\
\hline
\end{tabular}




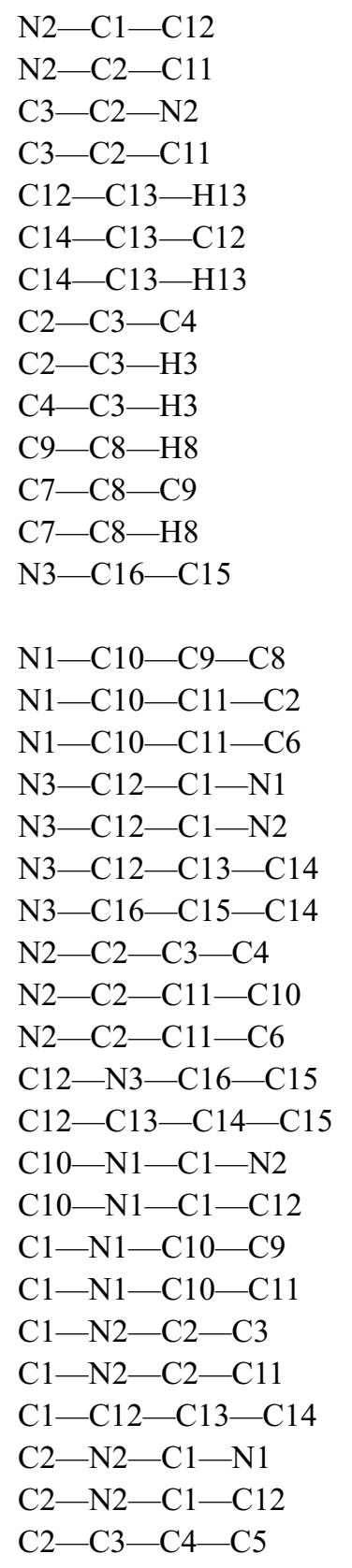

120.59 (14)

$120.58(14)$

$120.44(14)$

$118.98(15)$

$121.8(13)$

$118.23(15)$

119.9 (13)

$120.17(15)$

118.9 (12)

$120.9(12)$

$117.3(13)$

$121.68(15)$

$121.0(13)$

$123.42(15)$

$-179.50(15)$

$-1.0(2)$

$179.00(14)$

$1.1(2)$

-178.67 (14)

$-0.5(2)$

0.1 (3)

178.31 (14)

1.0 (2)

-179.07 (14)

$0.2(2)$

$0.8(2)$

-0.8 (2)

$179.41(13)$

$-178.72(15)$

$1.0(2)$

179.62 (14)

-0.7 (2)

179.19 (14)

0.7 (2)

$-179.58(13)$

$0.8(2)$
C4-C5-H5

$\mathrm{C} 13-\mathrm{C} 14-\mathrm{H} 14$

$\mathrm{C} 15-\mathrm{C} 14-\mathrm{C} 13$

C15-C14-H14

$\mathrm{C} 10-\mathrm{C} 11-\mathrm{C} 2$

$\mathrm{C} 10-\mathrm{C} 11-\mathrm{C} 6$

$\mathrm{C} 6-\mathrm{C} 11-\mathrm{C} 2$

$\mathrm{C} 8-\mathrm{C} 7-\mathrm{C} 6$

$\mathrm{C} 8-\mathrm{C} 7-\mathrm{H} 7$

$\mathrm{C} 6-\mathrm{C} 7-\mathrm{H} 7$

$\mathrm{C} 3-\mathrm{C} 4-\mathrm{H} 4$

$\mathrm{C} 5-\mathrm{C} 4-\mathrm{C} 3$

$\mathrm{C} 5-\mathrm{C} 4-\mathrm{H} 4$

$\mathrm{C} 13-\mathrm{C} 12-\mathrm{C} 1-\mathrm{N} 1$

$\mathrm{C} 13-\mathrm{C} 12-\mathrm{C} 1-\mathrm{N} 2$

$\mathrm{C} 3-\mathrm{C} 2-\mathrm{C} 11-\mathrm{C} 10$

$\mathrm{C} 3-\mathrm{C} 2-\mathrm{C} 11-\mathrm{C} 6$

$\mathrm{C} 16-\mathrm{N} 3-\mathrm{C} 12-\mathrm{C} 1$

$\mathrm{C} 16-\mathrm{N} 3-\mathrm{C} 12-\mathrm{C} 13$

$\mathrm{C} 16-\mathrm{C} 15-\mathrm{C} 14-\mathrm{C} 13$

$\mathrm{C} 9-\mathrm{C} 10-\mathrm{C} 11-\mathrm{C} 2$

$\mathrm{C} 9-\mathrm{C} 10-\mathrm{C} 11-\mathrm{C} 6$

$\mathrm{C} 9-\mathrm{C} 8-\mathrm{C} 7-\mathrm{C} 6$

$\mathrm{C} 6-\mathrm{C} 5-\mathrm{C} 4-\mathrm{C} 3$

$\mathrm{C} 5-\mathrm{C} 6-\mathrm{C} 11-\mathrm{C} 10$

$\mathrm{C} 5-\mathrm{C} 6-\mathrm{C} 11-\mathrm{C} 2$

$\mathrm{C} 5-\mathrm{C} 6-\mathrm{C} 7-\mathrm{C} 8$

$\mathrm{C} 11-\mathrm{C} 10-\mathrm{C} 9-\mathrm{C} 8$

$\mathrm{C} 11-\mathrm{C} 2-\mathrm{C} 3-\mathrm{C} 4$

$\mathrm{C} 11-\mathrm{C} 6-\mathrm{C} 5-\mathrm{C} 4$

$\mathrm{C} 11-\mathrm{C} 6-\mathrm{C} 7-\mathrm{C} 8$

$\mathrm{C} 7-\mathrm{C} 8-\mathrm{C} 9-\mathrm{C} 10$

$\mathrm{C} 7-\mathrm{C} 6-\mathrm{C} 5-\mathrm{C} 4$

$\mathrm{C} 7-\mathrm{C} 6-\mathrm{C} 11-\mathrm{C} 10$

$\mathrm{C} 7-\mathrm{C} 6-\mathrm{C} 11-\mathrm{C} 2$
$120.2(12)$

119.4 (14)

$119.24(15)$

121.4 (14)

$119.48(14)$

$119.78(14)$

$120.74(15)$

$120.58(15)$

120.3 (13)

119.2 (13)

$118.7(12)$

$121.57(15)$

119.8 (12)

$-178.61(14)$

$1.6(2)$

$-179.38(14)$

$0.6(2)$

$-179.71(13)$

$0.0(2)$

$-0.6(2)$

178.63 (15)

$-1.3(2)$

$-1.1(3)$

$0.5(2)$

$-179.34(14)$

0.7 (2)

$-179.48(16)$

$0.8(2)$

$-1.3(2)$

$-1.3(2)$

$0.6(2)$

$0.4(3)$

$178.83(16)$

$0.6(2)$

$-179.37(14)$

1-Methyl-2-(pyridin-2-yl)-1H-perimidine (2)

Crystal data

$\mathrm{C}_{17} \mathrm{H}_{13} \mathrm{~N}_{3}$

$M_{r}=259.30$

Monoclinic, $P 2_{1} / c$

$a=7.5095$ (2) $\AA$

$b=12.1216$ (3) $\AA$

$c=13.5616$ (4) $\AA$

$\beta=92.547(1)^{\circ}$

$V=1233.25(6) \AA^{3}$

$Z=4$

$F(000)=544$

$D_{\mathrm{x}}=1.397 \mathrm{Mg} \mathrm{m}^{-3}$

Mo $K \alpha$ radiation, $\lambda=0.71073 \AA$

Cell parameters from 5485 reflections

$\theta=2.3-30.5^{\circ}$ 
$\begin{aligned} \mu & =0.09 \mathrm{~mm}^{-1} \\ T & =100 \mathrm{~K}\end{aligned}$

\section{Data collection}

Bruker D8 Venture diffractometer

Radiation source: microfocus sealed X-ray tube, Incoatec $\mathrm{I} \mu \mathrm{S}$ microsource

Focusing mirrors monochromator

Detector resolution: 10.4 pixels $\mathrm{mm}^{-1}$

$\omega$-scan

Absorption correction: multi-scan

(SADABS; Krause et al., 2015)

\section{Refinement}

Refinement on $F^{2}$

Least-squares matrix: full

$R\left[F^{2}>2 \sigma\left(F^{2}\right)\right]=0.060$

$w R\left(F^{2}\right)=0.146$

$S=1.04$

3280 reflections

233 parameters

0 restraints
Block, orange

$0.34 \times 0.12 \times 0.11 \mathrm{~mm}$

$T_{\min }=0.685, T_{\max }=0.746$

14029 measured reflections

3280 independent reflections

2803 reflections with $I>2 \sigma(I)$

$R_{\text {int }}=0.035$

$\theta_{\text {max }}=29.0^{\circ}, \theta_{\min }=2.3^{\circ}$

$h=-10 \rightarrow 10$

$k=-15 \rightarrow 16$

$l=-18 \rightarrow 18$

Hydrogen site location: difference Fourier map

All $\mathrm{H}$-atom parameters refined

$w=1 /\left[\sigma^{2}\left(F_{\mathrm{o}}^{2}\right)+(0.0706 P)^{2}+0.7754 P\right]$

where $P=\left(F_{\mathrm{o}}^{2}+2 F_{\mathrm{c}}^{2}\right) / 3$

$(\Delta / \sigma)_{\max }<0.001$

$\Delta \rho_{\max }=0.45$ e $\AA^{-3}$

$\Delta \rho_{\min }=-0.33$ e $\AA^{-3}$

Special details

Geometry. All esds (except the esd in the dihedral angle between two 1.s. planes) are estimated using the full covariance matrix. The cell esds are taken into account individually in the estimation of esds in distances, angles and torsion angles; correlations between esds in cell parameters are only used when they are defined by crystal symmetry. An approximate (isotropic) treatment of cell esds is used for estimating esds involving l.s. planes.

Fractional atomic coordinates and isotropic or equivalent isotropic displacement parameters $\left(\AA^{2}\right)$

\begin{tabular}{lllll}
\hline & $x$ & $y$ & $z$ & $U_{\text {iso }} * / U_{\text {eq }}$ \\
\hline N2 & $0.76302(17)$ & $0.66381(10)$ & $0.66410(9)$ & $0.0173(3)$ \\
N3 & $0.79159(17)$ & $0.88961(10)$ & $0.52437(10)$ & $0.0197(3)$ \\
N1 & $0.69884(16)$ & $0.65068(10)$ & $0.49124(9)$ & $0.0153(3)$ \\
C11 & $0.79946(19)$ & $0.48774(12)$ & $0.57893(11)$ & $0.0160(3)$ \\
C1 & $0.71526(19)$ & $0.70642(12)$ & $0.57923(11)$ & $0.0155(3)$ \\
C13 & $0.68606(19)$ & $0.82886(12)$ & $0.58029(11)$ & $0.0157(3)$ \\
C2 & $0.80957(19)$ & $0.55209(12)$ & $0.66662(11)$ & $0.0166(3)$ \\
C14 & $0.5640(2)$ & $0.87417(13)$ & $0.64331(11)$ & $0.0177(3)$ \\
C6 & $0.85120(19)$ & $0.37474(12)$ & $0.58061(12)$ & $0.0187(3)$ \\
C12 & $0.6294(2)$ & $0.70168(13)$ & $0.39951(11)$ & $0.0194(3)$ \\
C3 & $0.50420(13)$ & $0.75469(12)$ & $0.0208(3)$ \\
C16 & $0.8700(2)$ & $1.05215(13)$ & $0.59611(12)$ & $0.0206(3)$ \\
C15 & $0.6670(2)$ & $0.98867(13)$ & $0.65099(12)$ & $0.0200(3)$ \\
C17 & $0.7802(2)$ & $0.99943(13)$ & $0.53330(13)$ & $0.0219(3)$ \\
C9 & $0.7297(2)$ & $0.47699(13)$ & $0.40235(12)$ & $0.0202(3)$ \\
C10 & $0.74095(19)$ & $0.53778(12)$ & $0.48835(11)$ & $0.0156(3)$ \\
C5 & $0.9117(2)$ & $0.32879(13)$ & $0.67236(13)$ & $0.0218(3)$ \\
C7 & $0.8416(2)$ & $0.31473(12)$ & $0.49048(13)$ & $0.0220(3)$
\end{tabular}




$\begin{array}{lllll}\text { C4 } & 0.9205(2) & 0.39232(13) & 0.75648(13) & 0.0226(3) \\ \text { C8 } & 0.7817(2) & 0.36497(13) & 0.40491(12) & 0.0228(3) \\ \text { H12A } & 0.603(3) & 0.7776(18) & 0.4103(15) & 0.029(5)^{*} \\ \text { H3 } & 0.879(3) & 0.5482(16) & 0.8163(15) & 0.027(5)^{*} \\ \text { H5 } & 0.951(3) & 0.2505(18) & 0.6727(15) & 0.030(5)^{*} \\ \text { H7 } & 0.882(3) & 0.2398(17) & 0.4889(14) & 0.025(5)^{*} \\ \text { H16 } & 0.663(3) & 1.1313(17) & 0.5991(14) & 0.026(5)^{*} \\ \text { H12B } & 0.723(3) & 0.7008(16) & 0.3497(14) & 0.023(5)^{*} \\ \text { H4 } & 0.961(3) & 0.3617(18) & 0.8184(16) & 0.033(5)^{*} \\ \text { H17 } & 0.856(3) & 1.0433(17) & 0.4948(15) & 0.028(5)^{*} \\ \text { H9 } & 0.695(3) & 0.5092(17) & 0.3402(15) & 0.026(5)^{*} \\ \text { H12C } & 0.518(3) & 0.6600(16) & 0.3771(14) & 0.025(5)^{*} \\ \text { H15 } & 0.474(3) & 1.0204(17) & 0.6955(15) & 0.027(5)^{*} \\ \text { H14 } & 0.489(3) & 0.8271(16) & 0.6786(14) & 0.023(5)^{*} \\ \text { H8 } & 0.777(3) & 0.3237(17) & 0.3434(16) & 0.033(5)^{*}\end{array}$

Atomic displacement parameters $\left(\AA^{2}\right)$

\begin{tabular}{lllllll}
\hline & $U^{11}$ & $U^{22}$ & $U^{33}$ & $U^{12}$ & $U^{13}$ & $U^{23}$ \\
\hline $\mathrm{N} 2$ & $0.0187(6)$ & $0.0155(6)$ & $0.0178(6)$ & $0.0007(5)$ & $0.0024(5)$ & $0.0012(5)$ \\
$\mathrm{N} 3$ & $0.0193(6)$ & $0.0155(6)$ & $0.0248(7)$ & $0.0029(5)$ & $0.0045(5)$ & $0.0026(5)$ \\
$\mathrm{N} 1$ & $0.0172(6)$ & $0.0128(6)$ & $0.0160(6)$ & $0.0024(5)$ & $0.0005(5)$ & $0.0006(4)$ \\
C11 & $0.0123(6)$ & $0.0151(7)$ & $0.0208(7)$ & $-0.0008(5)$ & $0.0030(5)$ & $0.0023(5)$ \\
C1 & $0.0130(6)$ & $0.0144(6)$ & $0.0192(7)$ & $0.0011(5)$ & $0.0023(5)$ & $-0.0003(5)$ \\
C13 & $0.0146(6)$ & $0.0148(6)$ & $0.0174(7)$ & $0.0021(5)$ & $-0.0011(5)$ & $-0.0006(5)$ \\
C2 & $0.0142(6)$ & $0.0158(7)$ & $0.0200(7)$ & $0.0003(5)$ & $0.0030(5)$ & $0.0025(5)$ \\
C14 & $0.0162(7)$ & $0.0194(7)$ & $0.0175(7)$ & $0.0007(6)$ & $0.0009(5)$ & $-0.0003(6)$ \\
C6 & $0.0143(6)$ & $0.0147(7)$ & $0.0274(8)$ & $-0.0006(5)$ & $0.0045(6)$ & $0.0027(6)$ \\
C12 & $0.0207(7)$ & $0.0182(7)$ & $0.0189(7)$ & $0.0038(6)$ & $-0.0024(6)$ & $0.0013(6)$ \\
C3 & $0.0200(7)$ & $0.0217(8)$ & $0.0206(7)$ & $-0.0001(6)$ & $0.0017(6)$ & $0.0040(6)$ \\
C16 & $0.0174(7)$ & $0.0143(7)$ & $0.0299(8)$ & $0.0027(6)$ & $-0.0020(6)$ & $-0.0023(6)$ \\
C15 & $0.0184(7)$ & $0.0216(7)$ & $0.0199(7)$ & $0.0046(6)$ & $-0.0006(6)$ & $-0.0045(6)$ \\
C17 & $0.0188(7)$ & $0.0167(7)$ & $0.0304(8)$ & $0.0010(6)$ & $0.0030(6)$ & $0.0035(6)$ \\
C9 & $0.0205(7)$ & $0.0184(7)$ & $0.0219(7)$ & $-0.0017(6)$ & $0.0026(6)$ & $-0.0019(6)$ \\
C10 & $0.0135(6)$ & $0.0131(6)$ & $0.0205(7)$ & $-0.0004(5)$ & $0.0033(5)$ & $-0.0004(5)$ \\
C5 & $0.0181(7)$ & $0.0144(7)$ & $0.0329(8)$ & $0.0011(6)$ & $0.0035(6)$ & $0.0076(6)$ \\
C7 & $0.0217(7)$ & $0.0118(7)$ & $0.0330(8)$ & $-0.0003(6)$ & $0.0076(6)$ & $-0.0015(6)$ \\
C4 & $0.0191(7)$ & $0.0223(8)$ & $0.0262(8)$ & $-0.0001(6)$ & $0.0010(6)$ & $0.0097(6)$ \\
C8 & $0.0234(8)$ & $0.0190(7)$ & $0.0265(8)$ & $-0.0037(6)$ & $0.0066(6)$ & $-0.0070(6)$ \\
& & & & & & \\
\hline
\end{tabular}

Geometric parameters $\left(\AA,{ }^{\circ}\right)$

\begin{tabular}{llll}
\hline $\mathrm{N} 2-\mathrm{C} 1$ & $1.2974(19)$ & $\mathrm{C} 12-\mathrm{H} 12 \mathrm{~B}$ & $0.996(19)$ \\
$\mathrm{N} 2-\mathrm{C} 2$ & $1.3986(18)$ & $\mathrm{C} 12-\mathrm{H} 12 \mathrm{C}$ & $1.01(2)$ \\
$\mathrm{N} 3-\mathrm{C} 13$ & $1.341(2)$ & $\mathrm{C} 3-\mathrm{C} 4$ & $1.408(2)$ \\
$\mathrm{N} 3-\mathrm{C} 17$ & $1.340(2)$ & $\mathrm{C} 3-\mathrm{H} 3$ & $0.99(2)$ \\
$\mathrm{N} 1-\mathrm{C} 1$ & $1.3720(18)$ & $\mathrm{C} 16-\mathrm{C} 15$ & $1.381(2)$ \\
$\mathrm{N} 1-\mathrm{C} 12$ & $1.4644(18)$ & $\mathrm{C} 16-\mathrm{C} 17$ & $1.386(2)$
\end{tabular}




\begin{tabular}{|c|c|c|c|}
\hline $\mathrm{N} 1-\mathrm{C} 10$ & $1.4055(18)$ & $\mathrm{C} 16-\mathrm{H} 16$ & $0.96(2)$ \\
\hline $\mathrm{C} 11-\mathrm{C} 2$ & $1.421(2)$ & C15-H15 & $0.96(2)$ \\
\hline $\mathrm{C} 11-\mathrm{C} 6$ & $1.424(2)$ & C17-H17 & $0.95(2)$ \\
\hline $\mathrm{C} 11-\mathrm{C} 10$ & $1.422(2)$ & $\mathrm{C} 9-\mathrm{C} 10$ & $1.379(2)$ \\
\hline $\mathrm{C} 1-\mathrm{C} 13$ & $1.5005(19)$ & $\mathrm{C} 9-\mathrm{C} 8$ & $1.413(2)$ \\
\hline $\mathrm{C} 13-\mathrm{C} 14$ & $1.393(2)$ & C9- $\mathrm{H} 9$ & $0.95(2)$ \\
\hline $\mathrm{C} 2-\mathrm{C} 3$ & $1.386(2)$ & $\mathrm{C} 5-\mathrm{C} 4$ & $1.376(2)$ \\
\hline $\mathrm{C} 14-\mathrm{C} 15$ & $1.394(2)$ & $\mathrm{C} 5-\mathrm{H} 5$ & $0.99(2)$ \\
\hline C14-H14 & $0.94(2)$ & $\mathrm{C} 7-\mathrm{C} 8$ & $1.369(2)$ \\
\hline $\mathrm{C} 6-\mathrm{C} 5$ & $1.419(2)$ & $\mathrm{C} 7-\mathrm{H} 7$ & $0.96(2)$ \\
\hline $\mathrm{C} 6-\mathrm{C} 7$ & $1.421(2)$ & $\mathrm{C} 4-\mathrm{H} 4$ & $0.96(2)$ \\
\hline $\mathrm{C} 12-\mathrm{H} 12 \mathrm{~A}$ & $0.95(2)$ & $\mathrm{C} 8-\mathrm{H} 8$ & $0.97(2)$ \\
\hline $\mathrm{C} 1-\mathrm{N} 2-\mathrm{C} 2$ & $117.78(13)$ & $\mathrm{C} 2-\mathrm{C} 3-\mathrm{H} 3$ & $120.6(12)$ \\
\hline $\mathrm{C} 17-\mathrm{N} 3-\mathrm{C} 13$ & $116.91(13)$ & $\mathrm{C} 4-\mathrm{C} 3-\mathrm{H} 3$ & $119.6(12)$ \\
\hline $\mathrm{C} 1-\mathrm{N} 1-\mathrm{C} 12$ & $123.08(12)$ & $\mathrm{C} 15-\mathrm{C} 16-\mathrm{C} 17$ & $118.60(14)$ \\
\hline $\mathrm{C} 1-\mathrm{N} 1-\mathrm{C} 10$ & $119.48(12)$ & $\mathrm{C} 15-\mathrm{C} 16-\mathrm{H} 16$ & $120.9(12)$ \\
\hline $\mathrm{C} 10-\mathrm{N} 1-\mathrm{C} 12$ & $117.35(12)$ & $\mathrm{C} 17-\mathrm{C} 16-\mathrm{H} 16$ & $120.4(12)$ \\
\hline $\mathrm{C} 2-\mathrm{C} 11-\mathrm{C} 6$ & $120.68(13)$ & $\mathrm{C} 14-\mathrm{C} 15-\mathrm{H} 15$ & $118.8(12)$ \\
\hline $\mathrm{C} 2-\mathrm{C} 11-\mathrm{C} 10$ & $119.45(13)$ & $\mathrm{C} 16-\mathrm{C} 15-\mathrm{C} 14$ & $118.81(14)$ \\
\hline $\mathrm{C} 10-\mathrm{C} 11-\mathrm{C} 6$ & $119.86(14)$ & $\mathrm{C} 16-\mathrm{C} 15-\mathrm{H} 15$ & $122.3(12)$ \\
\hline $\mathrm{N} 2-\mathrm{C} 1-\mathrm{N} 1$ & $125.88(13)$ & $\mathrm{N} 3-\mathrm{C} 17-\mathrm{C} 16$ & $123.87(15)$ \\
\hline $\mathrm{N} 2-\mathrm{C} 1-\mathrm{C} 13$ & $114.84(13)$ & N3-C17-H17 & $117.6(12)$ \\
\hline $\mathrm{N} 1-\mathrm{C} 1-\mathrm{C} 13$ & $119.20(12)$ & $\mathrm{C} 16-\mathrm{C} 17-\mathrm{H} 17$ & $118.5(12)$ \\
\hline $\mathrm{N} 3-\mathrm{C} 13-\mathrm{C} 1$ & $116.58(13)$ & $\mathrm{C} 10-\mathrm{C} 9-\mathrm{C} 8$ & $119.10(15)$ \\
\hline $\mathrm{N} 3-\mathrm{C} 13-\mathrm{C} 14$ & $123.48(14)$ & $\mathrm{C} 10-\mathrm{C} 9-\mathrm{H} 9$ & $122.2(12)$ \\
\hline $\mathrm{C} 14-\mathrm{C} 13-\mathrm{C} 1$ & $119.74(13)$ & $\mathrm{C} 8-\mathrm{C} 9-\mathrm{H} 9$ & $118.6(12)$ \\
\hline $\mathrm{N} 2-\mathrm{C} 2-\mathrm{C} 11$ & $120.45(13)$ & $\mathrm{N} 1-\mathrm{C} 10-\mathrm{C} 11$ & $116.90(13)$ \\
\hline $\mathrm{C} 3-\mathrm{C} 2-\mathrm{N} 2$ & $119.91(14)$ & $\mathrm{C} 9-\mathrm{C} 10-\mathrm{N} 1$ & $122.59(13)$ \\
\hline $\mathrm{C} 3-\mathrm{C} 2-\mathrm{C} 11$ & $119.62(14)$ & $\mathrm{C} 9-\mathrm{C} 10-\mathrm{C} 11$ & $120.51(14)$ \\
\hline $\mathrm{C} 13-\mathrm{C} 14-\mathrm{C} 15$ & $118.27(14)$ & $\mathrm{C} 6-\mathrm{C} 5-\mathrm{H} 5$ & $117.5(12)$ \\
\hline $\mathrm{C} 13-\mathrm{C} 14-\mathrm{H} 14$ & $119.6(12)$ & $\mathrm{C} 4-\mathrm{C} 5-\mathrm{C} 6$ & $120.59(14)$ \\
\hline $\mathrm{C} 15-\mathrm{C} 14-\mathrm{H} 14$ & $122.2(12)$ & $\mathrm{C} 4-\mathrm{C} 5-\mathrm{H} 5$ & $121.9(12)$ \\
\hline $\mathrm{C} 5-\mathrm{C} 6-\mathrm{C} 11$ & $117.91(14)$ & $\mathrm{C} 6-\mathrm{C} 7-\mathrm{H} 7$ & $120.0(12)$ \\
\hline $\mathrm{C} 5-\mathrm{C} 6-\mathrm{C} 7$ & $123.71(14)$ & $\mathrm{C} 8-\mathrm{C} 7-\mathrm{C} 6$ & $120.24(14)$ \\
\hline $\mathrm{C} 7-\mathrm{C} 6-\mathrm{C} 11$ & $118.37(14)$ & $\mathrm{C} 8-\mathrm{C} 7-\mathrm{H} 7$ & $119.7(12)$ \\
\hline $\mathrm{N} 1-\mathrm{C} 12-\mathrm{H} 12 \mathrm{~A}$ & $110.2(12)$ & $\mathrm{C} 3-\mathrm{C} 4-\mathrm{H} 4$ & $117.7(13)$ \\
\hline $\mathrm{N} 1-\mathrm{C} 12-\mathrm{H} 12 \mathrm{~B}$ & $109.7(11)$ & $\mathrm{C} 5-\mathrm{C} 4-\mathrm{C} 3$ & $121.47(15)$ \\
\hline $\mathrm{N} 1-\mathrm{C} 12-\mathrm{H} 12 \mathrm{C}$ & $107.6(11)$ & $\mathrm{C} 5-\mathrm{C} 4-\mathrm{H} 4$ & $120.9(13)$ \\
\hline $\mathrm{H} 12 \mathrm{~A}-\mathrm{C} 12-\mathrm{H} 12 \mathrm{~B}$ & 105.7 (17) & $\mathrm{C} 9-\mathrm{C} 8-\mathrm{H} 8$ & $118.3(12)$ \\
\hline $\mathrm{H} 12 \mathrm{~A}-\mathrm{C} 12-\mathrm{H} 12 \mathrm{C}$ & 110.7 (17) & $\mathrm{C} 7-\mathrm{C} 8-\mathrm{C} 9$ & $121.90(14)$ \\
\hline $\mathrm{H} 12 \mathrm{~B}-\mathrm{C} 12-\mathrm{H} 12 \mathrm{C}$ & $113.0(15)$ & $\mathrm{C} 7-\mathrm{C} 8-\mathrm{H} 8$ & $119.8(12)$ \\
\hline $\mathrm{C} 2-\mathrm{C} 3-\mathrm{C} 4$ & $119.73(15)$ & & \\
\hline $\mathrm{N} 2-\mathrm{C} 1-\mathrm{C} 13-\mathrm{N} 3$ & $118.34(15)$ & $\mathrm{C} 6-\mathrm{C} 11-\mathrm{C} 2-\mathrm{C} 3$ & $-0.2(2)$ \\
\hline $\mathrm{N} 2-\mathrm{C} 1-\mathrm{C} 13-\mathrm{C} 14$ & $-56.73(19)$ & $\mathrm{C} 6-\mathrm{C} 11-\mathrm{C} 10-\mathrm{N} 1$ & $-177.46(12)$ \\
\hline $\mathrm{N} 2-\mathrm{C} 2-\mathrm{C} 3-\mathrm{C} 4$ & $-178.61(13)$ & $\mathrm{C} 6-\mathrm{C} 11-\mathrm{C} 10-\mathrm{C} 9$ & $1.6(2)$ \\
\hline $\mathrm{N} 3-\mathrm{C} 13-\mathrm{C} 14-\mathrm{C} 15$ & $-2.1(2)$ & $\mathrm{C} 6-\mathrm{C} 5-\mathrm{C} 4-\mathrm{C} 3$ & $0.1(2)$ \\
\hline
\end{tabular}




$\begin{array}{llll}\mathrm{N} 1-\mathrm{C} 1-\mathrm{C} 13-\mathrm{N} 3 & -58.57(18) & \mathrm{C} 6-\mathrm{C} 7-\mathrm{C} 8-\mathrm{C} 9 & 0.7(2) \\ \mathrm{N} 1-\mathrm{C} 1-\mathrm{C} 13-\mathrm{C} 14 & 126.37(15) & \mathrm{C} 12-\mathrm{N} 1-\mathrm{C} 1-\mathrm{N} 2 & 174.50(14) \\ \mathrm{C} 11-\mathrm{C} 2-\mathrm{C} 3-\mathrm{C} 4 & -0.1(2) & \mathrm{C} 12-\mathrm{N} 1-\mathrm{C} 1-\mathrm{C} 13 & -9.0(2) \\ \mathrm{C} 11-\mathrm{C} 6-\mathrm{C} 5-\mathrm{C} 4 & -0.3(2) & \mathrm{C} 12-\mathrm{N} 1-\mathrm{C} 10-\mathrm{C} 11 & -176.81(13) \\ \mathrm{C} 11-\mathrm{C} 6-\mathrm{C} 7-\mathrm{C} 8 & -0.8(2) & \mathrm{C} 12-\mathrm{N} 1-\mathrm{C} 10-\mathrm{C} 9 & 4.2(2) \\ \mathrm{C} 1-\mathrm{N} 2-\mathrm{C} 2-\mathrm{C} 11 & -1.7(2) & \mathrm{C} 15-\mathrm{C} 16-\mathrm{C} 17-\mathrm{N} 3 & -2.0(2) \\ \mathrm{C} 1-\mathrm{N} 2-\mathrm{C} 2-\mathrm{C} 3 & 176.77(14) & \mathrm{C} 17-\mathrm{N} 3-\mathrm{C} 13-\mathrm{C} 1 & -172.77(13) \\ \mathrm{C} 1-\mathrm{N} 1-\mathrm{C} 10-\mathrm{C} 11 & -0.12(19) & \mathrm{C} 17-\mathrm{N} 3-\mathrm{C} 13-\mathrm{C} 14 & 2.1(2) \\ \mathrm{C} 1-\mathrm{N} 1-\mathrm{C} 10-\mathrm{C} 9 & -179.15(14) & \mathrm{C} 17-\mathrm{C} 16-\mathrm{C} 15-\mathrm{C} 14 & 1.9(2) \\ \mathrm{C} 1-\mathrm{C} 13-\mathrm{C} 14-\mathrm{C} 15 & 172.63(13) & \mathrm{C} 10-\mathrm{N} 1-\mathrm{C} 1-\mathrm{N} 2 & -2.0(2) \\ \mathrm{C} 13-\mathrm{N} 3-\mathrm{C} 17-\mathrm{C} 16 & 0.0(2) & \mathrm{C} 10-\mathrm{N} 1-\mathrm{C} 1-\mathrm{C} 13 & 174.54(12) \\ \mathrm{C} 13-\mathrm{C} 14-\mathrm{C} 15-\mathrm{C} 16 & 0.0(2) & \mathrm{C} 10-\mathrm{C} 11-\mathrm{C} 2-\mathrm{N} 2 & -0.2(2) \\ \mathrm{C} 2-\mathrm{N} 2-\mathrm{C} 1-\mathrm{N} 1 & 2.9(2) & \mathrm{C} 10-\mathrm{C} 11-\mathrm{C} 2-\mathrm{C} 3 & -178.68(13) \\ \mathrm{C} 2-\mathrm{N} 2-\mathrm{C} 1-\mathrm{C} 13 & -173.79(12) & \mathrm{C} 10-\mathrm{C} 11-\mathrm{C} 6-\mathrm{C} 5 & 178.90(13) \\ \mathrm{C} 2-\mathrm{C} 11-\mathrm{C} 6-\mathrm{C} 5 & 0.4(2) & \mathrm{C} 10-\mathrm{C} 11-\mathrm{C} 6-\mathrm{C} 7 & -0.3(2) \\ \mathrm{C} 2-\mathrm{C} 11-\mathrm{C} 6-\mathrm{C} 7 & -178.86(13) & \mathrm{C} 10-\mathrm{C} 9-\mathrm{C} 8-\mathrm{C} 7 & 0.6(2) \\ \mathrm{C} 2-\mathrm{C} 11-\mathrm{C} 10-\mathrm{N} 1 & 1.1(2) & \mathrm{C} 5-\mathrm{C} 6-\mathrm{C} 7-\mathrm{C} 8 & -179.97(15) \\ \mathrm{C} 2-\mathrm{C} 11-\mathrm{C} 10-\mathrm{C} 9 & -179.87(13) & \mathrm{C} 7-\mathrm{C} 6-\mathrm{C} 5-\mathrm{C} 4 & 178.87(15) \\ \mathrm{C} 2-\mathrm{C} 3-\mathrm{C} 4-\mathrm{C} 5 & 0.2(2) & \mathrm{C} 8-\mathrm{C} 9-\mathrm{C} 10-\mathrm{N} 1 & 177.31(13) \\ \mathrm{C} 6-\mathrm{C} 11-\mathrm{C} 2-\mathrm{N} 2 & 178.32(13) & \mathrm{C} 8-\mathrm{C} 9-\mathrm{C} 10-\mathrm{C} 11 & -1.7(2) \\ & & & \end{array}$

1,3-Dimethyl-2-(pyridin-2-yl)-1H-perimidinium iodide (3)

Crystal data

$\mathrm{C}_{18} \mathrm{H}_{16} \mathrm{~N}_{3}^{+} \cdot \mathrm{I}^{-}$

$M_{r}=401.24$

Monoclinic, $P 2_{1} / n$

$a=9.8821(2) \AA$

$b=9.7125(2) \AA$

$c=17.9839(4) \AA$

$\beta=103.676(1)^{\circ}$

$V=1677.15(6) \AA^{3}$

$Z=4$

\section{Data collection}

Bruker SMART APEXII

diffractometer

Radiation source: fine-focus sealed X-ray tube, X-ray tube

Mirror optics monochromator

Detector resolution: 7.9 pixels $\mathrm{mm}^{-1}$

$\omega$ scan

Absorption correction: multi-scan

(SADABS; Krause et al., 2015)

Refinement

Refinement on $F^{2}$

Least-squares matrix: full

$R\left[F^{2}>2 \sigma\left(F^{2}\right)\right]=0.028$

$w R\left(F^{2}\right)=0.067$

$S=1.06$

4146 reflections
$F(000)=792$

$D_{\mathrm{x}}=1.589 \mathrm{Mg} \mathrm{m}^{-3}$

Mo $K \alpha$ radiation, $\lambda=0.71073 \AA$

Cell parameters from 9859 reflections

$\theta=2.3-28.3^{\circ}$

$\mu=1.91 \mathrm{~mm}^{-1}$

$T=230 \mathrm{~K}$

Block, yellow

$0.32 \times 0.18 \times 0.13 \mathrm{~mm}$

$T_{\min }=0.668, T_{\max }=0.746$

28389 measured reflections

4146 independent reflections

3700 reflections with $I>2 \sigma(I)$

$R_{\text {int }}=0.026$

$\theta_{\max }=28.4^{\circ}, \theta_{\min }=2.3^{\circ}$

$h=-13 \rightarrow 13$

$k=-12 \rightarrow 12$

$l=-23 \rightarrow 23$

201 parameters

0 restraints

Primary atom site location: dual

Hydrogen site location: inferred from neighbouring sites

$\mathrm{H}$-atom parameters constrained 
$w=1 /\left[\sigma^{2}\left(F_{\mathrm{o}}^{2}\right)+(0.0273 P)^{2}+1.1509 P\right]$

where $P=\left(F_{\mathrm{o}}^{2}+2 F_{\mathrm{c}}{ }^{2}\right) / 3$

$(\Delta / \sigma)_{\max }<0.001$

$$
\Delta \rho_{\max }=0.72 \text { e } \AA^{-3}
$$

$\Delta \rho_{\min }=-0.46$ e $\AA^{-3}$

Special details

Geometry. All esds (except the esd in the dihedral angle between two 1.s. planes) are estimated using the full covariance matrix. The cell esds are taken into account individually in the estimation of esds in distances, angles and torsion angles; correlations between esds in cell parameters are only used when they are defined by crystal symmetry. An approximate (isotropic) treatment of cell esds is used for estimating esds involving 1.s. planes.

Fractional atomic coordinates and isotropic or equivalent isotropic displacement parameters $\left(\AA^{2}\right)$

\begin{tabular}{|c|c|c|c|c|}
\hline & $x$ & $y$ & $z$ & $U_{\text {iso }} * / U_{\text {eq }}$ \\
\hline I1 & $0.46311(2)$ & $0.43924(2)$ & $0.27610(2)$ & $0.05057(7)$ \\
\hline N2 & $0.70014(19)$ & $0.6145(2)$ & $0.48068(10)$ & $0.0417(4)$ \\
\hline $\mathrm{C} 14$ & $0.6404(2)$ & $0.8060(2)$ & $0.39271(12)$ & $0.0389(4)$ \\
\hline N1 & $0.78491(17)$ & $0.61380(19)$ & $0.37060(10)$ & $0.0380(4)$ \\
\hline $\mathrm{C} 11$ & $0.8424(2)$ & $0.4240(2)$ & $0.45912(13)$ & 0.0397 (4) \\
\hline $\mathrm{C} 15$ & $0.5033(2)$ & $0.8101(2)$ & $0.35210(14)$ & $0.0475(5)$ \\
\hline H15 & 0.453130 & 0.728579 & 0.336938 & $0.057^{*}$ \\
\hline $\mathrm{C} 1$ & $0.7119(2)$ & $0.6712(2)$ & $0.41527(12)$ & $0.0377(4)$ \\
\hline N3 & $0.7182(2)$ & $0.9164(2)$ & $0.41510(15)$ & $0.0633(6)$ \\
\hline $\mathrm{C} 2$ & $0.7651(2)$ & $0.4869(2)$ & $0.50629(13)$ & $0.0425(5)$ \\
\hline $\mathrm{C} 10$ & $0.8527(2)$ & $0.4851(2)$ & $0.38939(12)$ & $0.0401(4)$ \\
\hline $\mathrm{C} 3$ & $0.7545(3)$ & $0.4271(3)$ & $0.57385(16)$ & $0.0573(6)$ \\
\hline $\mathrm{H} 3$ & 0.701812 & 0.469174 & 0.604759 & $0.069^{*}$ \\
\hline C6 & $0.9113(2)$ & $0.2971(2)$ & $0.48227(15)$ & $0.0494(5)$ \\
\hline $\mathrm{C} 16$ & $0.4421(3)$ & $0.9371(3)$ & $0.33438(17)$ & $0.0562(6)$ \\
\hline $\mathrm{H} 16$ & 0.348626 & 0.943924 & 0.307067 & $0.067^{*}$ \\
\hline $\mathrm{C} 7$ & 0.9857 (3) & $0.2348(3)$ & $0.43274(18)$ & $0.0619(7)$ \\
\hline $\mathrm{H} 7$ & 1.031867 & 0.150768 & 0.446700 & $0.074 *$ \\
\hline $\mathrm{C} 13$ & $0.6248(3)$ & $0.6850(3)$ & $0.53130(15)$ & $0.0618(7)$ \\
\hline H13A & 0.589318 & 0.772451 & 0.508734 & $0.093^{*}$ \\
\hline H13B & 0.687651 & 0.701041 & 0.580789 & $0.093 *$ \\
\hline $\mathrm{H} 13 \mathrm{C}$ & 0.547719 & 0.628024 & 0.537689 & $0.093 *$ \\
\hline $\mathrm{C} 8$ & $0.9909(3)$ & $0.2948(3)$ & $0.36572(18)$ & $0.0646(7)$ \\
\hline $\mathrm{H} 8$ & 1.039315 & 0.250527 & 0.333392 & $0.078^{*}$ \\
\hline $\mathrm{C} 17$ & $0.5194(3)$ & $1.0531(2)$ & $0.35717(18)$ & $0.0600(7)$ \\
\hline H17 & 0.480165 & 1.141064 & 0.346141 & $0.072 *$ \\
\hline $\mathrm{C} 12$ & $0.7915(3)$ & $0.6767(3)$ & $0.29709(13)$ & $0.0525(6)$ \\
\hline $\mathrm{H} 12 \mathrm{~A}$ & 0.741265 & 0.619574 & 0.255397 & $0.079 *$ \\
\hline H12B & 0.887977 & 0.684564 & 0.294207 & $0.079^{*}$ \\
\hline $\mathrm{H} 12 \mathrm{C}$ & 0.749586 & 0.767565 & 0.293224 & $0.079 *$ \\
\hline C18 & $0.6552(4)$ & $1.0379(3)$ & $0.3964(2)$ & $0.0725(9)$ \\
\hline H18 & 0.707770 & 1.118127 & 0.411245 & $0.087^{*}$ \\
\hline $\mathrm{C} 9$ & $0.9255(3)$ & $0.4220(3)$ & $0.34300(16)$ & $0.0536(6)$ \\
\hline H9 & 0.931871 & 0.462969 & 0.296594 & $0.064^{*}$ \\
\hline $\mathrm{C} 5$ & $0.8993(3)$ & $0.2393(3)$ & $0.55276(18)$ & $0.0622(7)$ \\
\hline H5 & 0.944762 & 0.155928 & 0.569505 & $0.075^{*}$ \\
\hline
\end{tabular}




$\begin{array}{lllll}\mathrm{C} 4 & 0.8238(3) & 0.3020(3) & 0.59604(17) & 0.0659(8) \\ \mathrm{H} 4 & 0.817190 & 0.261112 & 0.642411 & 0.079^{*}\end{array}$

Atomic displacement parameters $\left(\AA^{2}\right)$

\begin{tabular}{lllllll}
\hline & $U^{11}$ & $U^{22}$ & $U^{33}$ & $U^{12}$ & $U^{13}$ & $U^{23}$ \\
\hline $\mathrm{I} 1$ & $0.05271(10)$ & $0.04098(9)$ & $0.05737(11)$ & $-0.00419(6)$ & $0.01171(7)$ & $-0.01150(6)$ \\
$\mathrm{N} 2$ & $0.0404(9)$ & $0.0444(10)$ & $0.0404(9)$ & $0.0032(8)$ & $0.0100(7)$ & $0.0044(8)$ \\
C14 & $0.0395(10)$ & $0.0339(10)$ & $0.0430(10)$ & $-0.0019(8)$ & $0.0090(8)$ & $0.0019(8)$ \\
N1 & $0.0339(8)$ & $0.0395(9)$ & $0.0389(9)$ & $-0.0004(7)$ & $0.0054(7)$ & $0.0043(7)$ \\
C11 & $0.0312(9)$ & $0.0350(10)$ & $0.0467(11)$ & $-0.0059(8)$ & $-0.0029(8)$ & $0.0020(8)$ \\
C15 & $0.0396(11)$ & $0.0363(11)$ & $0.0629(14)$ & $-0.0025(9)$ & $0.0049(10)$ & $0.0001(10)$ \\
C1 & $0.0313(9)$ & $0.0375(10)$ & $0.0416(10)$ & $-0.0021(8)$ & $0.0035(8)$ & $0.0033(8)$ \\
N3 & $0.0528(12)$ & $0.0445(12)$ & $0.0820(16)$ & $-0.0107(10)$ & $-0.0054(11)$ & $-0.0028(11)$ \\
C2 & $0.0363(10)$ & $0.0416(11)$ & $0.0463(11)$ & $-0.0045(9)$ & $0.0031(9)$ & $0.0085(9)$ \\
C10 & $0.0326(10)$ & $0.0401(11)$ & $0.0427(11)$ & $-0.0011(8)$ & $-0.0005(8)$ & $-0.0030(9)$ \\
C3 & $0.0531(14)$ & $0.0652(17)$ & $0.0532(14)$ & $-0.0032(12)$ & $0.0118(11)$ & $0.0165(12)$ \\
C6 & $0.0384(11)$ & $0.0356(11)$ & $0.0644(14)$ & $-0.0056(9)$ & $-0.0072(10)$ & $0.0028(10)$ \\
C16 & $0.0457(13)$ & $0.0501(14)$ & $0.0697(16)$ & $0.0107(11)$ & $0.0079(12)$ & $0.0046(12)$ \\
C7 & $0.0487(14)$ & $0.0378(12)$ & $0.088(2)$ & $0.0056(11)$ & $-0.0068(13)$ & $-0.0064(12)$ \\
C13 & $0.0679(16)$ & $0.0709(17)$ & $0.0523(14)$ & $0.0169(14)$ & $0.0255(12)$ & $0.0077(13)$ \\
C8 & $0.0539(15)$ & $0.0597(16)$ & $0.0761(18)$ & $0.0122(13)$ & $0.0072(13)$ & $-0.0189(14)$ \\
C17 & $0.0728(18)$ & $0.0353(12)$ & $0.0735(17)$ & $0.0086(11)$ & $0.0201(14)$ & $0.0032(11)$ \\
C12 & $0.0540(13)$ & $0.0605(15)$ & $0.0452(12)$ & $0.0073(12)$ & $0.0163(10)$ & $0.0136(11)$ \\
C18 & $0.075(2)$ & $0.0353(13)$ & $0.099(2)$ & $-0.0130(13)$ & $0.0047(17)$ & $-0.0059(13)$ \\
C9 & $0.0497(13)$ & $0.0563(14)$ & $0.0530(13)$ & $0.0056(11)$ & $0.0086(11)$ & $-0.0080(11)$ \\
C5 & $0.0538(14)$ & $0.0416(13)$ & $0.0792(18)$ & $-0.0041(11)$ & $-0.0085(13)$ & $0.0203(12)$ \\
C4 & $0.0599(16)$ & $0.0647(17)$ & $0.0673(17)$ & $-0.0064(14)$ & $0.0036(13)$ & $0.0311(14)$ \\
& & & & & &
\end{tabular}

Geometric parameters $\left(A,{ }^{\circ}\right)$

\begin{tabular}{llll}
\hline $\mathrm{N} 2-\mathrm{C} 1$ & $1.328(3)$ & $\mathrm{C} 6-\mathrm{C} 5$ & $1.417(4)$ \\
$\mathrm{N} 2-\mathrm{C} 2$ & $1.422(3)$ & $\mathrm{C} 16-\mathrm{H} 16$ & 0.9400 \\
$\mathrm{~N} 2-\mathrm{C} 13$ & $1.474(3)$ & $\mathrm{C} 16-\mathrm{C} 17$ & $1.369(4)$ \\
$\mathrm{C} 14-\mathrm{C} 15$ & $1.379(3)$ & $\mathrm{C} 7-\mathrm{H} 7$ & 0.9400 \\
$\mathrm{C} 14-\mathrm{C} 1$ & $1.497(3)$ & $\mathrm{C} 7-\mathrm{C} 8$ & $1.351(4)$ \\
$\mathrm{C} 14-\mathrm{N} 3$ & $1.326(3)$ & $\mathrm{C} 13-\mathrm{H} 13 \mathrm{~A}$ & 0.9700 \\
$\mathrm{~N} 1-\mathrm{C} 1$ & $1.323(3)$ & $\mathrm{C} 13-\mathrm{H} 13 \mathrm{~B}$ & 0.9700 \\
$\mathrm{~N} 1-\mathrm{C} 10$ & $1.421(3)$ & $\mathrm{C} 13-\mathrm{H} 13 \mathrm{C}$ & 0.9700 \\
$\mathrm{~N} 1-\mathrm{C} 12$ & $1.472(3)$ & $\mathrm{C} 8-\mathrm{H} 8$ & 0.9400 \\
$\mathrm{C} 11-\mathrm{C} 2$ & $1.408(3)$ & $\mathrm{C} 8-\mathrm{C} 9$ & $1.409(4)$ \\
$\mathrm{C} 11-\mathrm{C} 10$ & $1.413(3)$ & $\mathrm{C} 17-\mathrm{H} 17$ & 0.9400 \\
$\mathrm{C} 11-\mathrm{C} 6$ & $1.422(3)$ & $\mathrm{C} 17-\mathrm{C} 18$ & $1.368(4)$ \\
$\mathrm{C} 15-\mathrm{H} 15$ & 0.9400 & $\mathrm{C} 12-\mathrm{H} 12 \mathrm{~A}$ & 0.9700 \\
$\mathrm{C} 15-\mathrm{C} 16$ & $1.378(3)$ & $\mathrm{C} 12-\mathrm{H} 12 \mathrm{~B}$ & 0.9700 \\
$\mathrm{~N} 3-\mathrm{C} 18$ & $1.339(4)$ & $\mathrm{C} 12-\mathrm{H} 12 \mathrm{C}$ & 0.9700 \\
$\mathrm{C} 2-\mathrm{C} 3$ & $1.373(3)$ & $\mathrm{C} 18-\mathrm{H} 18$ & 0.9400 \\
$\mathrm{C} 10-\mathrm{C} 9$ & $1.369(3)$ & $\mathrm{C} 9-\mathrm{H} 9$ & 0.9400
\end{tabular}




\begin{tabular}{|c|c|c|c|}
\hline $\mathrm{C} 3-\mathrm{H} 3$ & 0.9400 & $\mathrm{C} 5-\mathrm{H} 5$ & 0.9400 \\
\hline $\mathrm{C} 3-\mathrm{C} 4$ & $1.405(4)$ & $\mathrm{C} 5-\mathrm{C} 4$ & $1.345(4)$ \\
\hline $\mathrm{C} 6-\mathrm{C} 7$ & $1.417(4)$ & $\mathrm{C} 4-\mathrm{H} 4$ & 0.9400 \\
\hline $\mathrm{C} 1-\mathrm{N} 2-\mathrm{C} 2$ & $121.39(19)$ & $\mathrm{C} 6-\mathrm{C} 7-\mathrm{H} 7$ & 119.6 \\
\hline $\mathrm{C} 1-\mathrm{N} 2-\mathrm{C} 13$ & $121.1(2)$ & $\mathrm{C} 8-\mathrm{C} 7-\mathrm{C} 6$ & $120.8(2)$ \\
\hline $\mathrm{C} 2-\mathrm{N} 2-\mathrm{C} 13$ & $117.40(19)$ & $\mathrm{C} 8-\mathrm{C} 7-\mathrm{H} 7$ & 119.6 \\
\hline $\mathrm{C} 15-\mathrm{C} 14-\mathrm{C} 1$ & $120.71(19)$ & $\mathrm{N} 2-\mathrm{C} 13-\mathrm{H} 13 \mathrm{~A}$ & 109.5 \\
\hline $\mathrm{N} 3-\mathrm{C} 14-\mathrm{C} 15$ & $124.3(2)$ & $\mathrm{N} 2-\mathrm{C} 13-\mathrm{H} 13 \mathrm{~B}$ & 109.5 \\
\hline $\mathrm{N} 3-\mathrm{C} 14-\mathrm{C} 1$ & $114.99(19)$ & $\mathrm{N} 2-\mathrm{C} 13-\mathrm{H} 13 \mathrm{C}$ & 109.5 \\
\hline $\mathrm{C} 1-\mathrm{N} 1-\mathrm{C} 10$ & $121.36(18)$ & $\mathrm{H} 13 \mathrm{~A}-\mathrm{C} 13-\mathrm{H} 13 \mathrm{~B}$ & 109.5 \\
\hline $\mathrm{C} 1-\mathrm{N} 1-\mathrm{C} 12$ & $121.10(18)$ & $\mathrm{H} 13 \mathrm{~A}-\mathrm{C} 13-\mathrm{H} 13 \mathrm{C}$ & 109.5 \\
\hline $\mathrm{C} 10-\mathrm{N} 1-\mathrm{C} 12$ & $117.43(18)$ & $\mathrm{H} 13 \mathrm{~B}-\mathrm{C} 13-\mathrm{H} 13 \mathrm{C}$ & 109.5 \\
\hline $\mathrm{C} 2-\mathrm{C} 11-\mathrm{C} 10$ & $121.08(19)$ & $\mathrm{C} 7-\mathrm{C} 8-\mathrm{H} 8$ & 119.2 \\
\hline $\mathrm{C} 2-\mathrm{C} 11-\mathrm{C} 6$ & $119.4(2)$ & $\mathrm{C} 7-\mathrm{C} 8-\mathrm{C} 9$ & $121.7(3)$ \\
\hline $\mathrm{C} 10-\mathrm{C} 11-\mathrm{C} 6$ & $119.6(2)$ & $\mathrm{C} 9-\mathrm{C} 8-\mathrm{H} 8$ & 119.2 \\
\hline $\mathrm{C} 14-\mathrm{C} 15-\mathrm{H} 15$ & 120.9 & $\mathrm{C} 16-\mathrm{C} 17-\mathrm{H} 17$ & 120.8 \\
\hline $\mathrm{C} 16-\mathrm{C} 15-\mathrm{C} 14$ & $118.2(2)$ & $\mathrm{C} 18-\mathrm{C} 17-\mathrm{C} 16$ & $118.4(2)$ \\
\hline $\mathrm{C} 16-\mathrm{C} 15-\mathrm{H} 15$ & 120.9 & $\mathrm{C} 18-\mathrm{C} 17-\mathrm{H} 17$ & 120.8 \\
\hline $\mathrm{N} 2-\mathrm{C} 1-\mathrm{C} 14$ & $117.88(19)$ & $\mathrm{N} 1-\mathrm{C} 12-\mathrm{H} 12 \mathrm{~A}$ & 109.5 \\
\hline $\mathrm{N} 1-\mathrm{C} 1-\mathrm{N} 2$ & $122.52(19)$ & $\mathrm{N} 1-\mathrm{C} 12-\mathrm{H} 12 \mathrm{~B}$ & 109.5 \\
\hline $\mathrm{N} 1-\mathrm{C} 1-\mathrm{C} 14$ & $119.60(18)$ & $\mathrm{N} 1-\mathrm{C} 12-\mathrm{H} 12 \mathrm{C}$ & 109.5 \\
\hline $\mathrm{C} 14-\mathrm{N} 3-\mathrm{C} 18$ & $115.8(2)$ & $\mathrm{H} 12 \mathrm{~A}-\mathrm{C} 12-\mathrm{H} 12 \mathrm{~B}$ & 109.5 \\
\hline $\mathrm{C} 11-\mathrm{C} 2-\mathrm{N} 2$ & $116.78(19)$ & $\mathrm{H} 12 \mathrm{~A}-\mathrm{C} 12-\mathrm{H} 12 \mathrm{C}$ & 109.5 \\
\hline $\mathrm{C} 3-\mathrm{C} 2-\mathrm{N} 2$ & $122.2(2)$ & $\mathrm{H} 12 \mathrm{~B}-\mathrm{C} 12-\mathrm{H} 12 \mathrm{C}$ & 109.5 \\
\hline $\mathrm{C} 3-\mathrm{C} 2-\mathrm{C} 11$ & $121.0(2)$ & $\mathrm{N} 3-\mathrm{C} 18-\mathrm{C} 17$ & $124.5(2)$ \\
\hline $\mathrm{C} 11-\mathrm{C} 10-\mathrm{N} 1$ & $116.85(19)$ & N3-C18-H18 & 117.8 \\
\hline $\mathrm{C} 9-\mathrm{C} 10-\mathrm{N} 1$ & $122.4(2)$ & $\mathrm{C} 17-\mathrm{C} 18-\mathrm{H} 18$ & 117.8 \\
\hline $\mathrm{C} 9-\mathrm{C} 10-\mathrm{C} 11$ & $120.8(2)$ & $\mathrm{C} 10-\mathrm{C} 9-\mathrm{C} 8$ & $119.1(3)$ \\
\hline $\mathrm{C} 2-\mathrm{C} 3-\mathrm{H} 3$ & 120.6 & $\mathrm{C} 10-\mathrm{C} 9-\mathrm{H} 9$ & 120.4 \\
\hline $\mathrm{C} 2-\mathrm{C} 3-\mathrm{C} 4$ & $118.9(3)$ & $\mathrm{C} 8-\mathrm{C} 9-\mathrm{H} 9$ & 120.4 \\
\hline $\mathrm{C} 4-\mathrm{C} 3-\mathrm{H} 3$ & 120.6 & $\mathrm{C} 6-\mathrm{C} 5-\mathrm{H} 5$ & 119.6 \\
\hline $\mathrm{C} 7-\mathrm{C} 6-\mathrm{C} 11$ & $118.0(2)$ & $\mathrm{C} 4-\mathrm{C} 5-\mathrm{C} 6$ & $120.9(2)$ \\
\hline $\mathrm{C} 5-\mathrm{C} 6-\mathrm{C} 11$ & $118.0(2)$ & $\mathrm{C} 4-\mathrm{C} 5-\mathrm{H} 5$ & 119.6 \\
\hline $\mathrm{C} 5-\mathrm{C} 6-\mathrm{C} 7$ & $123.9(2)$ & $\mathrm{C} 3-\mathrm{C} 4-\mathrm{H} 4$ & 119.1 \\
\hline $\mathrm{C} 15-\mathrm{C} 16-\mathrm{H} 16$ & 120.5 & $\mathrm{C} 5-\mathrm{C} 4-\mathrm{C} 3$ & $121.8(3)$ \\
\hline $\mathrm{C} 17-\mathrm{C} 16-\mathrm{C} 15$ & $118.9(2)$ & $\mathrm{C} 5-\mathrm{C} 4-\mathrm{H} 4$ & 119.1 \\
\hline $\mathrm{C} 17-\mathrm{C} 16-\mathrm{H} 16$ & 120.5 & & \\
\hline $\mathrm{N} 2-\mathrm{C} 2-\mathrm{C} 3-\mathrm{C} 4$ & $-178.8(2)$ & $\mathrm{C} 2-\mathrm{C} 11-\mathrm{C} 6-\mathrm{C} 5$ & $-0.2(3)$ \\
\hline $\mathrm{C} 14-\mathrm{C} 15-\mathrm{C} 16-\mathrm{C} 17$ & $0.4(4)$ & $\mathrm{C} 2-\mathrm{C} 3-\mathrm{C} 4-\mathrm{C} 5$ & $-0.3(4)$ \\
\hline $\mathrm{C} 14-\mathrm{N} 3-\mathrm{C} 18-\mathrm{C} 17$ & $0.3(5)$ & $\mathrm{C} 10-\mathrm{N} 1-\mathrm{C} 1-\mathrm{N} 2$ & $1.7(3)$ \\
\hline $\mathrm{N} 1-\mathrm{C} 10-\mathrm{C} 9-\mathrm{C} 8$ & $179.6(2)$ & $\mathrm{C} 10-\mathrm{N} 1-\mathrm{C} 1-\mathrm{C} 14$ & $-179.10(18)$ \\
\hline $\mathrm{C} 11-\mathrm{C} 2-\mathrm{C} 3-\mathrm{C} 4$ & $0.7(4)$ & $\mathrm{C} 10-\mathrm{C} 11-\mathrm{C} 2-\mathrm{N} 2$ & $-0.7(3)$ \\
\hline $\mathrm{C} 11-\mathrm{C} 10-\mathrm{C} 9-\mathrm{C} 8$ & $-0.2(4)$ & $\mathrm{C} 10-\mathrm{C} 11-\mathrm{C} 2-\mathrm{C} 3$ & $179.7(2)$ \\
\hline $\mathrm{C} 11-\mathrm{C} 6-\mathrm{C} 7-\mathrm{C} 8$ & $0.2(4)$ & $\mathrm{C} 10-\mathrm{C} 11-\mathrm{C} 6-\mathrm{C} 7$ & $-1.6(3)$ \\
\hline $\mathrm{C} 11-\mathrm{C} 6-\mathrm{C} 5-\mathrm{C} 4$ & $0.6(4)$ & $\mathrm{C} 10-\mathrm{C} 11-\mathrm{C} 6-\mathrm{C} 5$ & $179.6(2)$ \\
\hline $\mathrm{C} 15-\mathrm{C} 14-\mathrm{C} 1-\mathrm{N} 2$ & $-87.1(3)$ & $\mathrm{C} 6-\mathrm{C} 11-\mathrm{C} 2-\mathrm{N} 2$ & $179.11(18)$ \\
\hline
\end{tabular}




\begin{tabular}{llll}
$\mathrm{C} 15-\mathrm{C} 14-\mathrm{C} 1-\mathrm{N} 1$ & $93.6(3)$ & $\mathrm{C} 6-\mathrm{C} 11-\mathrm{C} 2-\mathrm{C} 3$ & $-0.5(3)$ \\
$\mathrm{C} 15-\mathrm{C} 14-\mathrm{N} 3-\mathrm{C} 18$ & $0.6(4)$ & $\mathrm{C} 6-\mathrm{C} 11-\mathrm{C} 10-\mathrm{N} 1$ & $-178.17(18)$ \\
$\mathrm{C} 15-\mathrm{C} 16-\mathrm{C} 17-\mathrm{C} 18$ & $0.4(5)$ & $\mathrm{C} 6-\mathrm{C} 11-\mathrm{C} 10-\mathrm{C} 9$ & $1.6(3)$ \\
$\mathrm{C} 1-\mathrm{N} 2-\mathrm{C} 2-\mathrm{C} 11$ & $0.2(3)$ & $\mathrm{C} 6-\mathrm{C} 7-\mathrm{C} 8-\mathrm{C} 9$ & $1.2(4)$ \\
$\mathrm{C} 1-\mathrm{N} 2-\mathrm{C} 2-\mathrm{C} 3$ & $179.7(2)$ & $\mathrm{C} 6-\mathrm{C} 5-\mathrm{C} 4-\mathrm{C} 3$ & $-0.4(4)$ \\
$\mathrm{C} 1-\mathrm{C} 14-\mathrm{C} 15-\mathrm{C} 16$ & $178.6(2)$ & $\mathrm{C} 16-\mathrm{C} 17-\mathrm{C} 18-\mathrm{N} 3$ & $-0.8(5)$ \\
$\mathrm{C} 1-\mathrm{C} 14-\mathrm{N} 3-\mathrm{C} 18$ & $-179.0(3)$ & $\mathrm{C} 7-\mathrm{C} 6-\mathrm{C} 5-\mathrm{C} 4$ & $-178.1(3)$ \\
$\mathrm{C} 1-\mathrm{N} 1-\mathrm{C} 10-\mathrm{C} 11$ & $-2.1(3)$ & $\mathrm{C} 7-\mathrm{C} 8-\mathrm{C} 9-\mathrm{C} 10$ & $-1.3(4)$ \\
$\mathrm{C} 1-\mathrm{N} 1-\mathrm{C} 10-\mathrm{C} 9$ & $178.1(2)$ & $\mathrm{C} 13-\mathrm{N} 2-\mathrm{C} 1-\mathrm{C} 14$ & $-2.8(3)$ \\
$\mathrm{N} 3-\mathrm{C} 14-\mathrm{C} 15-\mathrm{C} 16$ & $-1.0(4)$ & $\mathrm{C} 13-\mathrm{N} 2-\mathrm{C} 1-\mathrm{N} 1$ & $176.4(2)$ \\
$\mathrm{N} 3-\mathrm{C} 14-\mathrm{C} 1-\mathrm{N} 2$ & $92.5(3)$ & $\mathrm{C} 13-\mathrm{N} 2-\mathrm{C} 2-\mathrm{C} 11$ & $-177.0(2)$ \\
$\mathrm{N} 3-\mathrm{C} 14-\mathrm{C} 1-\mathrm{N} 1$ & $-86.8(3)$ & $\mathrm{C} 13-\mathrm{N} 2-\mathrm{C} 2-\mathrm{C} 3$ & $2.6(3)$ \\
$\mathrm{C} 2-\mathrm{N} 2-\mathrm{C} 1-\mathrm{C} 14$ & $-179.88(18)$ & $\mathrm{C} 12-\mathrm{N} 1-\mathrm{C} 1-\mathrm{N} 2$ & $177.7(2)$ \\
$\mathrm{C} 2-\mathrm{N} 2-\mathrm{C} 1-\mathrm{N} 1$ & $-0.6(3)$ & $\mathrm{C} 12-\mathrm{N} 1-\mathrm{C} 1-\mathrm{C} 14$ & $-3.1(3)$ \\
$\mathrm{C} 2-\mathrm{C} 11-\mathrm{C} 10-\mathrm{N} 1$ & $1.7(3)$ & $\mathrm{C} 12-\mathrm{N} 1-\mathrm{C} 10-\mathrm{C} 11$ & $-178.33(19)$ \\
$\mathrm{C} 2-\mathrm{C} 11-\mathrm{C} 10-\mathrm{C} 9$ & $-178.6(2)$ & $\mathrm{C} 12-\mathrm{N} 1-\mathrm{C} 10-\mathrm{C} 9$ & $1.9(3)$ \\
$\mathrm{C} 2-\mathrm{C} 11-\mathrm{C} 6-\mathrm{C} 7$ & $178.6(2)$ & $\mathrm{C} 5-\mathrm{C} 6-\mathrm{C} 7-\mathrm{C} 8$ & $178.9(3)$ \\
\hline
\end{tabular}

